\title{
FTO Regulates Microglia-Induced Inflammation by Stabilizing ADAM17 Expression After Experimental Traumatic Brain Injury
}

\section{Xiangrong Chen ( $\nabla$ xiangrong_chen281@126.com )}

Second Affiliated Hospital of Fujian Medical University https://orcid.org/0000-0001-9400-7358

Jieran Yao

Second Affiliated Hospital of Fujian Medical University

\section{Yue Chen}

First Affiliated Hospital of Fujian Medical University

\section{Wenqi Lv}

First Affiliated Hospital of Fujian Medical University

\section{Yuanxiang Lin}

First Affiliated Hospital of Fujian Medical University

\section{Wenhua Fang}

First Affiliated Hospital of Fujian Medical University

Jinhui Chen

First Affiliated Hospital of Fujian Medical University

\section{Dezhi Kang}

First Affiliated Hospital of Fujian Medical University

\section{Chenyu Ding}

First Affiliated Hospital of Fujian Medical University

\section{Research}

Keywords: Traumatic brain injury, Microglia, Neuroinflammation囚m6A, FTO, ADAM17

Posted Date: January 5th, 2022

DOI: https://doi.org/10.21203/rs.3.rs-1134958/v1

License: (c) (i) This work is licensed under a Creative Commons Attribution 4.0 International License. Read Full License 


\section{Abstract \\ Background}

The neuroinflammatory response mediated by microglial polarization plays an important role in the secondary nerve injury of traumatic brain injury (TBI). The post-transcriptional modification of n6methyladenosine $\left(\mathrm{m}^{6} \mathrm{~A}\right)$ is ubiquitous in the immune response of the central nervous system. The fat mass and obesity (FTO)-related protein can regulate the splicing process of pre-mRNA. However, after experimental traumatic brain injury (TBI), the role of FTO in microglial polarization and the subsequent neuroinflammatory response is still unclear.

\section{Methods}

TBI mice model was established by the Feeney weight-drop method. Neurological severity score, brain water content measurement and Nissl staining were used to detect the role of FTO in microglial polarization and the molecular mechanism of targeted RNA epigenetic modification. In vitro and in vivo experiments were conducted to evaluate microglial polarization and the neuroinflammatory response by down-regulation of FTO expression. The level of $\mathrm{m}^{6} \mathrm{~A}$ modification in $\mathrm{M} 1$ activated microglia was detected by qRT-PCR, $\mathrm{m}^{6} \mathrm{~A}-\mathrm{MeRIP}$ and $\mathrm{m}^{6} \mathrm{~A}$ high-throughput sequencing. Fluorescent in situ hybridization combined with immunofluorescence imaging were used to detect the epigenetic regulation of ADAM17 mediated by an FTO-m ${ }^{6} \mathrm{~A}$-dependent mechanism.

\section{Results}

The expression of FTO was significantly down-regulated in BV2 cells treated with lipopolysaccharide and mice with TBI. Down-regulation of FTO expression increased the level of $m^{6} \mathrm{~A}$ in $\mathrm{M} 1$ microglia at the level of the entire transcriptome. Meanwhile, after FTO interference, $\mathrm{M} 1 / \mathrm{M} 0$ phenotype detection experiments revealed the BV2 cells shifted from an M0 to M1 phenotype as the population rate of $\mathrm{CD} 11 \mathrm{~b}^{+} / \mathrm{CD} 86^{+}$ increased and secretion of pro-inflammatory cytokines was enhanced. Methylated RNA immunoprecipitation assay showed that the $\mathrm{m}^{6} \mathrm{~A}$ peaks located in the ADAM17 and TNF-a genes increased. Taken together, the results indicated that FTO can affect the transcription modification of ADAM17 and the expression of the downstream TNF-a/NF-kB pathway. In turn, ADAM17 can block the $\mathrm{M} 1$-phenotypic transition of microglia driven by FTO- $\mathrm{m}^{6} \mathrm{~A}$ modification.

\section{Conclusions}

The down-regulation of FTO expression leads to the abnormally high expression of ADAM17 in microglia. The activation of microglia and neuroinflammatory response regulated by FTO-related $\mathrm{m}^{6} \mathrm{~A}$ modification play an important role in the early pro-inflammatory process of TBI secondary injury. 


\section{Significance Statement}

Microglia-mediated neuroinflammatory responses play important roles in secondary neurological injury after TBI. Regulation of the phenotype of microglia and inflammatory factors is a potential strategy for the treatment of TBI. The fat mass and obesity (FTO)-related protein can regulate the splicing process of pre-mRNA. However, after TBI, the role of FTO in microglial polarization and the subsequent neuroinflammatory response is still unclear. Here, we report that the expression of FTO was significantly down-regulated in BV2 cells treated with lipopolysaccharide and mice with TBI. FTO can affect the transcription modification of ADAM17 and downstream TNF-a/NF-kB pathway related factors in microglia, which promote $\mathrm{M} 1$ polarization phenotype of microglia and the development of inflammation after TBI. Taken together, our results reveal that FTO, as an important $\mathrm{m}^{6} \mathrm{~A}$ demethylation enzyme, regulates microglia polarization and neuroinflammation by stabilizing ADAM17 expression after TBI.

\section{Introduction}

Traumatic brain injury (TBI) is a common serious disease, with high fatality and disability rates. TBI is associated with a primary injury that triggers a series of harmful inflammatory processes that further aggravate initial tissue damage and affect nerve cell repair [1-3]. Microglia are the innate immune cells of the central nervous system, which play an important role in neuroinflammation and neurological impairment after TBI [4]. The activation of microglia is closely related to their different polarization phenotypes. Specifically, microglia activated by traumatic stress exhibit two polarized phenotypes, including the classic activated M1 phenotype and the alternative activated M2 phenotype, which are also called the pro-inflammatory phenotype and anti-inflammatory phenotype, respectively [5-7]. Our previous studies $[8,9]$ confirmed that the polarization phenotype of microglia is closely related to the outcome of neuroinflammation. Blocking polarization of the M1 phenotype and adjusting the M1/M2 polarization can improve the prognosis of neuroinflammation after TBI and restore nerve function.

A disintegrin and metalloproteases (ADAMs) are metalloendopeptidases [10]. In the ADAMs family, ADAM17 (also known as tumor necrosis factor-a converting enzyme [11]) and its close relative, ADAM10, are types of transmembrane metalloproteases [11-13]. It has been reported that these two metalloendopeptidases mediate pro-inflammatory cytokine expression [11, 12]. In particular, TNF-a, TGFa, and bidirectional regulators are cleaved by ADAM17 sheddase activity, while EGF and $\beta$ cell proteins are cleaved by ADAM10 [11, 12]. Indeed, it has been shown that the ADAM17/TNF-a/NF-kB pathway plays an important role in initiating and regulating polarization of macrophages and the inflammatory cascade [14-16].

The eukaryotic RNA modification n6-methyladenosine $\left(\mathrm{m}^{6} \mathrm{~A}\right)$ has recently been identified as a key posttranscriptional regulator of gene expression [17-19]. $\mathrm{M}^{6} \mathrm{~A}$ mRNA modification is the most abundant type of gene modification, accounting for more than $60 \%$ of all post-transcriptional RNA modifications [17]. Previous studies have reported that the modification of $\mathrm{m}^{6} \mathrm{~A}$ mRNA mainly involves the modification of adenine sites, and which was precisely determined by writer, eraser, and reader proteins in the RNA 
RRACH consensus motif ( $R=G$ or $A, H=A$ or $U$ ) [20-22]. $M^{6} A$ modification is involved in the epigenetic regulation of multiple biological processes such as neurodevelopment, immune response, and DNA damage repair. It also plays a broad role in the stability, localization, transportation, splicing, and translation of mRNA and RNA [21, 23-25].

The fat mass and obesity (FTO) associated gene is a $58 \mathrm{kDa}$ member of the ALKB family of ferrous/2oxoglutarate-dependent oxidative DNA/RNA demethylases [26-28]. FTO, as a demethylase, may affect the RNA binding ability of the shear factor SRSF2, thereby reversing the methylation modification of the RRACH sequence, and further regulating the shearing process of pre-mRNA [28, 29]. Abnormal FTO regulation is related to obesity [29], brain nerve development [26], and neurotransmission [27], suggesting that $\mathrm{m}^{6} \mathrm{~A}$ modification may play an important regulatory role in many human neurological diseases. Epitranscriptomic profiling of $\mathrm{m}^{6} \mathrm{~A}$ methylation after TBI in the mouse cortex indicated that FTO might be an intervention target of TBI epigenetic modification [30]. However, whether $\mathrm{m}^{6} \mathrm{~A}$ demethylase FTO affects microglia-mediated neuroinflammation and its mechanisms remain unclear.

This study aimed to explore the role of FTO in microglial polarization and the molecular mechanism of targeted RNA epigenetic modification. We demonstrated that down-regulation of FTO expression leads to abnormally high expression of ADAM17 in microglia after TBI, thereby promoting the development of neuroinflammation. Targeted over-expression of ADAM17 significantly offset the anti-inflammatory effect of FTO-enhanced M1 microglial polarization.

\section{Methods}

\section{Animals}

Adult male C57BL/ 6 mice, 10 weeks old (weight 20-25 g), purchased from the Experimental Animal Center of Fujian Medical University, were raised in a clean temperature-controlled environment $\left(23 \pm 2{ }^{\circ} \mathrm{C}\right)$, with a $12 \mathrm{~h}$ light/dark cycle and with food and drink available ad libitum. The experimental protocol of this study, including all surgical operations and animal use, was approved by the Experimental Animal Ethics Committee of Fujian Medical University and carried out in accordance with the National Institutes of Health (Bethesda, MD, USA) "Guidelines for the Care and Use of Laboratory Animals".

\section{Animal experiment}

The mice were randomly divided into a Sham group, TBI group, TBI+Vehicle group, and TBI+NADP group $(n=24)$, and each group was divided into four subgroups (1-, 3-, 7-, and 14-day time points). The controlled cortical impact (CCl) injury model used in here was established as previously described [31, 32]. Anesthesia was administered in a Perspex container using $3 \%$ isoflurane delivered by a animal anesthetic machine (RWD Life Science Co., Shenzhen, China). Mice were then subjected to an impact using a $2 \mathrm{~mm}$ metal flat-tip impactor (Brain and Spinal Cord Impactor, 68099H, RWD Life Science). The center of impact was bregma $2.5 \mathrm{~mm}$ and lateral $2.5 \mathrm{~mm}$. The velocity was $5 \mathrm{~m} / \mathrm{s}$, depth $3 \mathrm{~mm}$, and impact speed $100 \mathrm{~ms}$. In the sham operation group, the surgery was performed but the impact was 
omitted. Mice in the TBI+NADP group were injected with NADP $(300 \mathrm{mg} / \mathrm{kg} /$ day; Sigma-Aldrich, St. Louis, MO, USA) $0.5 \mathrm{~h}$ after the surgery [33], those in the TBI+Vehicle group were given an equal volume of the vehicle dimethyl sulfoxide as a negative control.

\section{Neurological impairment score}

Mice were subjected to exercise (muscular phenotype and abnormal action), sensation (visual, tactile, and balance), and reflex examinations and assigned a modified neurological severity score (mNSS). A score was recorded when the mice failed to complete the task or showed no corresponding reflexes. The mNSS score ranged from 0 to 18 points, where a total score of 18 points indicated severe neurological deficits and a score of 0 indicated normal performance. Researchers, blinded to the experimental groups, measured the neurological function of mice at different time points.

\section{Measurement of brain water content and blood brain barrier (BBB) permeability}

The wet weight-dry weight method was used to calculate the brain water content ${ }^{8}$. The animals were sacrificed after neurological assessment, and the cerebral cortex was excised at the edge of the bone window. Filter paper was used to remove excess blood and cerebrospinal fluid. The wet weight was measured and the brains were dried in an oven for $24 \mathrm{~h}$ at $100{ }^{\circ} \mathrm{C}$ until a constant weight was achieved, at which point the dry weight was measured. The percentage of brain water content was calculated as (wet weight - dry weight)/wet weight $\times 100 \%$.

BBB permeability was investigated by measuring the extravasation of Evans blue dye ( $2 \%$ in saline; 4 $\mathrm{mL} / \mathrm{kg}$; Sigma-Aldrich), which was injected intravenously $2 \mathrm{~h}$ prior to sacrifice on the third day after injury. Following sacrifice, the mice were transcardially perfused with PBS followed by PBS containing 4\% paraformaldehyde. Each tissue sample was immediately weighed, homogenized in $1 \mathrm{~mL}$ of $50 \%$ trichloroacetic acid, and centrifuged. Then, the absorption of the supernatant was measured with a spectrophotometer (UV-1800 ENG 240V; Shimadzu Corpomiceion, Kyoto, Japan) at a wavelength of 620 $\mathrm{nm}$. The quantity of Evans blue dye was calculated using a standard curve and expressed as $\mu \mathrm{g} / \mathrm{g}$ of brain tissue.

\section{Nissl staining}

The formaldehyde-fixed specimens were embedded in paraffin, cut into $4 \mu \mathrm{m}$ thick sections, deparaffinized with xylene, and rehydrated in a graded series of alcohol. After being treated with Nissl staining solution for $5 \mathrm{~min}$, the damaged neurons were atrophied or contained vacuoles, while the cells of normal neurons were larger and fuller with larger nuclei. Five areas were randomly selected for microscopic examination by a researcher who was blinded to the to the experimental groups.

\section{Cell culture and treatment}

Mouse microglia and BV2 cells were provided by Basic Medical Sciences Chinese Academy of Medical Sciences (Beijing, China) and cultured at $37^{\circ} \mathrm{C}, 95 \% \mathrm{O}_{2}, 5 \% \mathrm{CO}_{2}$, in Dulbecco's Modified Eagle's Medium 
(KeyGEN Biotech,Nan jing, China) supplemented with 10\% fetal bovine serum (Gibco, USA) and 1\% penicillin/streptomycin (KeyGEN Biotech). BV2 cells were stimulated with $10 \mu \mathrm{g} / \mathrm{ml}$ LPS (Meilune, China) for $2 \mathrm{~h}$ to induce inflammation. The ADAM17 inhibitor TAPI-1 ( $1 \mu \mathrm{M}$ : MCE, China) was applied to the BV2 cell groups for downstream study of the $\mathrm{FTO} / \mathrm{m}^{6} \mathrm{~A} / \mathrm{ADAM} 17$ signaling pathway-mediated polarization.

\section{Cell transfection}

FTO siRNAs and plasmids were utilized to knock down (siFTO group) and overexpress FTO in BV2 cells (oeFTO group), to explore the function of FTO in regulating the microglial polarization. BV2 cells were transfected with FTO siRNA and pcDNA3.1-Flag-FTO, respectively. Mouse FTO siRNA and negative control were chemically synthesized by RiboBio (Guangzhou, China). The mouse pcDNA3.1-Flag-FTO plasmid and vector were obtained from Honorgene (Changsha, China). The sequence for the control siRNA was as follows: 5'-AAGGCUCUAUGAAGAGGCUTG-3'. The three sequences for the FTO siRNAs were as follows (FTO siRNA01-siRNA03): 5'-AGACCUUCCUAAAGCUCAAUG-3', 5'-GCACCUACAAGUACUUGAACA3 ' and 5'-CCAAAGAUGAUGAGUUCUAUC-3'. The FTO siRNA and FTO plasmid, and its corresponding negative control, were transiently transfected into BV2 cells with Lipofectamine 2000 for $24 \mathrm{~h}$ at $37^{\circ} \mathrm{C}$, according to the manufacturer's instructions and previous work [34,35]. After, the transfected BV2 cells were treated with the ADAM17 inhibitor, TAPI- $1(1 \mu \mathrm{M})$, for another $48 \mathrm{~h}$ at $37^{\circ} \mathrm{C}$ for signaling pathway assessment.

\section{RNA isolation and RT-PCR}

Total RNA from the tissues or the cultured samples was purified using TRIzol (Invitrogen, ThermoFisher Scientific) and reverse transcribed using the $A B I$ reverse transcriptase ( $A B I$, ThermoFisher Scientific), oligo (dT) primers, or specific RT primers. Template $(1 \mu \mathrm{L})$ was amplified by real-time PCR using the primers listed in Suppl. Table 4, Supporting Information (Integrated DNA Technologies). Each sample was run in triplicate in a $10 \mu$ reaction with $100 \mathrm{~nm}$ forward and reverse primers, $2 \mu$ l of SYBR Green mix (ABI, ThermoFisher Scientific), and $10 \mathrm{ng}$ cDNA. The PCR reactions were carried out using a STEP-ONE 96 realtime PCR system. GAPDH was used as an internal control for normalization. Ratios of mRNA levels from the treated groups or mRNA at levels different time points compared with the mRNA level of the normal control group were calculated using the $\Delta \mathrm{Ct}$ method $\left(2^{-\Delta \Delta \mathrm{Ct}}\right)$. All data were normalized to GAPDH.

\section{RNA $\mathrm{m}^{6} \mathrm{~A}$ quantification}

Total RNA was isolated with TRIzol (Invitrogen, ThermoFisher Scientific) according to the manufacturer's instructions and RNA quality was measured by using a NanoDrop3000. The $\mathrm{m}^{6} \mathrm{~A}$ RNA methylation quantification kit (Abcam, UK) was used to measure the $\mathrm{m}^{6} \mathrm{~A}$ content of the RNA. Briefly, $200 \mathrm{ng}$ of RNA was detected in each well. The capture antibody solution and detection antibody solution were then added to assay wells separately in a suitable diluted concentration following the manufacturer's instructions. The $\mathrm{m}^{6} \mathrm{~A}$ levels were quantified calorimetrically by reading the $\mathrm{OD}_{450}$ absorbance of each well and calculations were performed based on the standard curve. 


\section{RNA $m^{6} A$ sequence and $m^{6} A-R N A$ immunoprecipitation assay}

The chemically fragmented RNA (100 nucleotides) was incubated with the $\mathrm{m}^{6} \mathrm{~A}$ antibody and immunoprecipitation was performed according to the standard protocol of the Magna methylated RNA immune-precipitation (MeRIP) $\mathrm{m}^{6} \mathrm{~A}$ Kit (Merck Millipore, USA). Enrichment of $\mathrm{m}^{6} \mathrm{~A}$ containing mRNA was analyzed by qRT-PCR using the primers listed in Suppl. Table 5. For high-throughput sequencing, the RNA fragments purified from $\mathrm{m}^{6} \mathrm{~A}-\mathrm{MeRIP}$ were used for library construction with the NEBNext ${ }^{\circledR}$ Ultra ${ }^{\text {TM }}$ RNA Library Prep Kit (NEB, USA) and sequenced with an Illumina SE50. Sequencing reads were aligned to the mouse genome $\mathrm{mm} 9 / \mathrm{mm} 10$ by Bowtie2, and the $\mathrm{m}^{6} \mathrm{~A}$ peaks were detected by magnetic cell sorting as described [36].

\section{Peak annotation and motif identification}

MACS2 (version 2.1.0.20151222) was employed to identify $\mathrm{m}^{6} \mathrm{~A}$ peaks, after which Hommer (version: 4.8) was used to annotate the $\mathrm{m}^{6} \mathrm{~A}$ peaks. The nucleotides in $\mathrm{m}^{6} \mathrm{~A}$ peak regions were used for detection of the consensus $\mathrm{m}^{6} \mathrm{~A}$ motif by DREME (version: 4.11.1) and MEME (version: 4.11.1). Motif central enrichment was performed by CentriMo (version: 4.11.1). Differential methylation was determined by Pyicoenrich (version: 2.0.7).

\section{Luciferase reporter assays and mutagenesis assay}

The dual-luciferase vector pmigLO (Promega, USA) was used to identify the $\mathrm{m}^{6} \mathrm{~A}$ functional site of ADAM7. The CDS and 3'UTR regions of ADAM17 (NM_009615.6) were amplified by PCR using the genomic DNA from BV2 cells as a wild type template. Three putative $\mathrm{m}^{6} \mathrm{~A}$ recognition sites were identified in the CDS and 3'UTR. A QuikChange II XL Site-Directed Mutagenesis Kit (Agilent, USA) was used to generate a point mutation (alanine to thymine) according to the manufacturer's instructions. Luciferase activity was measured with a Dual Luciferase Reporter Gene Assay (Beyotime, China) on an MD SpectraMax M3 plate reader. Experiments were performed in triplicate. Data are presented as mean values $( \pm S D)$.

\section{Immunohistochemical analysis}

The formaldehyde-fixed specimens were embedded in paraffin, cut into $4 \mu \mathrm{m}$ thick sections, deparaffinized with xylene, and rehydrated in a graded series of alcohol. TAntigen retrieval was carried out by microwaving the sections in citric acid buffer. Sections were then incubated with an antibody against FTO (1:400, abcam, UK), washed, and then incubated with secondary antibody. The negative control was prepared without adding the primary antibody. Five randomly selected visual fields were analyzed as follows $[3,8,37]$ : 0 , no positive cells; 1 , very few positive cells; 2 , moderate number of positive cells; 3 , many positive cells; and 4 , the highest number of positive cells.

\section{Immunofluorescence analysis}


The formaldehyde-fixed specimens were embedded in paraffin, cut into $4 \mu \mathrm{m}$-thick sections, deparaffinized with xylene, rehydrated in a graded series of alcohol, and then the antigen was retrieved as describe above. Sections were incubated overnight at $4{ }^{\circ} \mathrm{C}$ with antibodies against ionized calciumbinding adapter molecule-1 (Iba-1; 1:200; Santa Cruz Biotechnology, Santa Cruz, CA, USA), FTO (1:200; Santa Cruz Biotechnology), CD86, and CD206 (1:100; Boster Biotech, Wuhan, China). After washing, the sections were incubated with secondary antibodies for $1 \mathrm{~h}$ at room temperature, after which the cell nuclei were stained with 4',6-diamidino-2-phenylindole. Immuno-positive cells in five randomly selected fields were counted under a microscope (Leica, Wetzlar, Germany) at $400 \times$ magnification by investigators who were blinded to the experimental groups.

\section{Fluorescent in situ hybridization (FISH) combined with immunofluorescence imaging}

The Cy3-labeled probes of ADAM17 mRNA were designed and synthesized by Sangon Inc. (Shanghai), while mouse monoclonal antibody to FTO and goat anti-mouse IgG H\&L were obtained from abcam (Alexa Fluor ${ }^{\circledR} 488$, abcam, UK). FISH combined with immunofluorescence experiments were performed according to the manufacturer's instructions. Bv2 cells were seeded in a 24-well plate on chamber cover slips and treated as described above. After the cells had reached $60 \%-70 \%$ confluency, they were fixed with $4 \%$ paraformaldehyde for $30 \mathrm{~min}$ at room temperature, permeabilized with pre-cooled $0.5 \%$ Triton-X100 for 5 min at $4{ }^{\circ} \mathrm{C}$, washed three times with PBS, and prehybridized for $30 \mathrm{~min}$ at $37^{\circ} \mathrm{C}$ with $200 \mu \mathrm{L}$ pre-hybridization buffer. mRNA ADAM17 FISH Probe Mix Storage solution $(2.5 \mu \mathrm{L}, 20 \mu \mathrm{M}$; mRNA FISH Probe Mix) and $100 \mu \mathrm{L}$ hybridization buffer were added and the cells were incubated overnight at $37^{\circ} \mathrm{C}$ in a humidified chamber in the dark. The cells were then washed three times for 5 min each with $4 \times$ SSC and $2 \times$ SSC for 5 min and $1 \times$ SSC for 5 min at $42^{\circ} \mathrm{C}$, followed by a 5 min wash with PBS at room temperature in the dark. Finally, glass coverslips were sealed with an anti-quenching adhesive containing DAPI and images were acquired on an IX51 inverted microscope (Olympus, Japan).

\section{Enzyme-linked immunosorbent assay (ELISA)}

Inflammatory factors in brain tissue and BV2 cell culture supernatant were detected using mouse ELISA kits (KeyGEN, China) for TNF- $\alpha$, IL-1 $\beta$, IL-6, TGF- $\beta 1$, and IFN- $\gamma$. The optical density at $450 \mathrm{~nm}$ was measured using a microplate reader (SpectraMax M3, Molecular Devices, Inc.).

\section{Western Blotting Analysis}

Samples, including brain tissues and BV2 cells, were prepared by using the nuclear and cytoplasmic proteins purification assay kit (KeyGEN Biotech, China), with modified RIPA lysis buffer (50mM Tris-HCl $\mathrm{pH} 7.4,150 \mathrm{mM} \mathrm{NaCl}, 1 \%$ Triton $\mathrm{X}-100,1 \%$ sodium deoxycholate, $0.1 \%$ SDS). The protein concentrations of the nuclear and cytosolic lysates, respectively, were determined with a BCA kit (Beyotime, China), and approximately $25 \mu \mathrm{g}$ of protein was loaded to each lane and transferred to polyvinylidene difluoride membranes. The membranes were then incubated with primary antibodies against $B$ cell lymphoma-2 (Bcl-2; 1:2000), Bcl-2-associated X factor (Bax; 1:5000), cleaved caspase-3 (1:3000), Iba-1 (1:1000), CD86, CD206, IL-1ß, iNOS, Arg-1 (1:2000), FTO (1:500), ADAM17 (1:1000), and TGF- $\beta 1$ (1:1000) (all purchased 
from Abcam). Immunoreactive bands were visualized with an ECL Western Blotting Detection Kit (Millipore, Billerica, MA, USA). Grey value analysis was conducted uisng UN-Scan-It 6.1 software (Silk Scientific Inc., Orem, UT, USA). The expression levels were normalized against $\beta$-actin (1:5000, Boster Biotech, China).

\section{Statistical analysis}

Data were analyzed using SPSS v.18.0 software (SPSS Inc., Chicago, IL, USA). All experiments were performed in triplicate unless otherwise noted, and the results are expressed as the mean \pm SD. The unpaired Student's t test was used for comparison between groups. Multiple-group comparisons were assessed by one-way ANOVA and post hoc multiple comparisons were performed using StudentNewman-Keuls tests. $P<0.05$ was considered statistically significant.

\section{Results}

\section{The level of $\mathrm{m}^{6} \mathrm{~A}$ modification is increased in $\mathrm{M} 1$ activated microglia}

In order to explore whether $\mathrm{m}^{6} \mathrm{~A}$ methylation was related to microglial polarization, we first investigated BV2 cells without any treatment (M0 phenotype group) and lipopolysaccharide (LPS)-stimulated BV2 cells (M1 phenotype group) to determine the abundance of $\mathrm{m}^{6} \mathrm{~A}$ and $\mathrm{m}^{6} \mathrm{~A}$ writers/erasers in the two groups. $\mathrm{m}^{6} \mathrm{~A}$ RNA methylation quantitative experiments revealed that, compared with the $\mathrm{M} 0$ phenotype, the level of $\mathrm{m}^{6} \mathrm{~A}$ modification in the M1 phenotype was significantly higher (Fig. 1a). RT-PCR results showed that the expressions of hnRNP, YTHDF1, and YTHDF2 increased in the M1 phenotype group compared with the M0 phenotype group, although the difference was not statistically significant. By contrast, FTO, WTAP, METTL3, and METTL14 decreased in the M1 phenotype group, with the FTO level showing a significant reduction $(P<0.01)$, and which was consistent with the dynamic change of the $\mathrm{m}^{6} \mathrm{~A}$ modification level (Fig. 1b). The expression of FTO protein was lower in the M1 phenotype group, but with no significant change in the expression of ALKBH5 (Fig. 1C). Furthermore, we used MeRIP-Seq to analyze the transcriptome-wide mRNA modifications in the M0 and M1 phenotype groups. Specifically, the clean reads of the two groups were compared with the mouse genome to obtain positional information of the reference genome (details in Suppl. Table 1). In the M0 and M1-phenotype groups, the transcripts of 4828 and 5767 genes were enriched with high-confidence $\mathrm{m}^{6} \mathrm{~A}$ peaks, respectively. Motif enrichment analysis revealed that the $\mathrm{m}^{6} \mathrm{~A}$ peaks identified above shared a common sequence element [U]GGAC[U]A (Fig. 1d) and that the CDS and 5'UTR, together with the $3^{\prime} \mathrm{UTR}$, harbored the largest fraction of peaks (Fig. 1e, f). Furthermore, the results showed that the $\mathrm{m}^{6} \mathrm{~A}$ modification level in the $\mathrm{M} 1$ phenotype group was significantly higher than that in the $\mathrm{M} 0$ phenotype group. Additionally, multiple $\mathrm{m}^{6} \mathrm{~A}$ peaks in the M1 phenotype group were enriched in transcripts of the phosphoinositide 3-kinase (PI3K)/Akt and NF$K B$ signaling pathways compared with the M0 phenotype group (Fig. $1 \mathrm{~g}, \mathrm{~h}$ ). These data indicated the potential role of $\mathrm{m}^{6} \mathrm{~A}$ in activating $\mathrm{M} 1$ microglia during the initial stages of inflammation.

\section{FTO regulates microglial M1 polarization}


Since FTO was down-regulated in the M1 phenotype group, we further verified the role of FTO in the polarization and activation of microglia. FTO siRNAs and plasmids were utilized to knock down and overexpress FTO in BV2 cells, respectively囚to explore the function of FTO in regulating the microglial polarization. The level of FTO mRNA was significantly reduced after siRNA treatment (siFTO group), and upregulated after pcDNA3.1-Flag-FTO transfection (oeFTO group) (Fig. 2a, b). As shown in Fig. 2c, under LPS stimulation, the M1 phenotype $(\mathrm{CD} 11 \mathrm{~b}+/ \mathrm{CD} 86+)$ in the siFTO group was higher $(\mathrm{P}<0.05)$ compared with the negative control and oeFTO groups. Furthermore, immunofluorescence and ELISA were used to detect alterations in the levels of inflammatory cytokines following both FTO knockdown and overexpression. The expression of anti-inflammatory factors (IL-10 and TGF- $\beta 1$ ) was found to be the opposite (Fig. $2 d-f$ ). These data suggest that the $\mathrm{m}^{6} \mathrm{~A}$ demethylase FTO inhibits M1 microglial polarization during the inflammatory response.

\section{ADAM17 is the downstream target of FTO-mediated $\mathrm{m}^{6} \mathrm{~A}$ modification in microglia}

Next, we investigated the molecular mechanism of FTO in regulating microglial polarization and identified downstream transcription targets. Compared with the control group, under LPS stimulation, 918 genes were altered, of which 532 genes were up-regulated and 386 genes were down-regulated (Fig. $3 a$ ). Using standard GEO2R analysis and quantile normalization, we chose 88 genes with significant changes in the two signaling pathways associated with the initial inflammatory response (Suppl. Table 2). In order to characterize the potential targets involved in the inflammatory response process of BV2 cells regulated by $\mathrm{m}^{6} \mathrm{~A}$, we identified 88 inflammatory response process genes with key functions. We overlapped the related genes with 19 genes from the $918 \mathrm{~m}^{6} \mathrm{~A}$ regulatory genes in the initial inflammatory response (> two-fold change of $\mathrm{m}^{6} \mathrm{~A}$, Fig. 3b), including TNF-a/NF-kB pathway genes (Fig. 3c). The TNF-a/NF-kB pathway is critical to the polarization of the M1 microglial phenotype $[8,38]$. The activity of TNF-a is closely associated with the extracellular domain cleaved by ADAM17 $[39,40]$. According to our analysis, $\mathrm{PI} 3 \mathrm{~K} / \mathrm{Akt}$ and TNF-a/NF-kB signaling pathway genes were enriched in the M1 phenotype group compared with the $\mathrm{MO}$ phenotype group, with several $\mathrm{m}^{6} \mathrm{~A}$ motifs that were enriched in $\mathrm{mRNA}$ transcripts including HMGB1, RELA, ADAM17, and TNF-a (Fig. 3d and Suppl. Table 3). The results indicated that ADAM17/TNF- $a$ /NF-kB pathway genes were not affected by FTO-mediated $\mathrm{m}^{6} \mathrm{~A}$ modifications. Moreover, compared with the siFTO group, both the mRNA and protein levels of ADAM17, TNF-a, and NF-KB decreased (Fig. 3e, f). Based on these data, we concluded that the down-regulation of FTO promoted the

expression of ADAM17 protein at the translation level by maintaining the stability of the ADAM17 mRNA transcript.

\section{Epigenetic regulation of ADAM17 is mediated by an FTO- ${ }^{6}$ A-dependent mechanism}

To verify the hypothesis that FTO promotes ADAM17 protein expression by enhancing the stability of the ADAM17 mRNA transcript, we used real time-PCR to detect the expression of the ADAM17 precursor and mature mRNA. As expected, compared with oeFTO group, both the ADAM17 precursor and mature mRNA in the siFTO group were significantly increased (Fig. 4a). Since the mRNA level depends on its 
transcription and stability, we carried out half-life detection by using actinomycin $D$ treatment and found that the ADAM17 precursor mRNA levels were similar in the FTO overexpression and siFTO groups; however, the mature ADAM17 mRNA levels in the siFTO group were significantly increased compared with those in the FTO overexpression group (Fig. 4b). Consistent with the above results, western blot analysis showed that when cells in the groups were treated with the protein translation inhibitor cycloheximide (CHX), the half-life of ADAM17 protein in cells of the siFTO group was longer than that in cells of the FTO overexpression group (Fig. 4c). The results suggest that FTO-m ${ }^{6} \mathrm{~A}$-modification of ADAM17 not only increases the translation level of ADAM17 protein by enhancing the stability of mature ADAM17 mRNA, but also enhances the stability of ADAM17 protein.

In order to prove that the $\mathrm{m}^{6} \mathrm{~A}$ modification site of ADAM17 mRNA was directly demethylated by FTO, we prepared multiple fragments of the ADAM17 transcript through in vitro mRNA transcription experiments, including the 5'UTR (1-200) CDS (201-2484), and 3' UTR (2489-4451) regions (Fig. 4d). We used sitedirected mutagenesis to replace the adenosine base in the $\mathrm{m}^{6} \mathrm{~A}$ consensus sequence of ADAM17 with thymine, thereby eliminating the three potential $\mathrm{m}^{6} \mathrm{~A}$ sites in the CDS and $3^{\prime} \mathrm{UTR}$ regions (RRACH) (Fig. $4 \mathrm{e})$. As a result, the mutant 03 of ADAM17 had markedly decreased luciferase activity compared with wild type ADAM17 when normalized to the Renilla data (Fig. 4f). These data demonstrated that FTOmediated $\mathrm{m}^{6} \mathrm{~A}$ demethylation accelerated the degradation of ADAM17 mRNA, while silencing of FTO enhanced the stability of ADAM17 mRNA, which in turn increased its protein expression and stability in an $\mathrm{m}^{6} \mathrm{~A}$ demethylase-dependent manner.

\section{Inhibition of ADAM17 blocks M1 microglial polarization driven by FTO-m ${ }^{6} \mathrm{~A}$-modifications}

To further verify the role of ADAM17 as a downstream target gene of FTO in microglial polarization, we explored the co-localization of FTO and ADAM17 mRNA in BV2 cells. Over-expression of FTO resulted in the degradation of ADAM17 RNA. Over-expression of FTO showed a strong signal of red fluorescence (FTO+) and a relatively weak signal of green fluorescence (ADAM17+), while down-regulation of FTO showed the opposite trend. In agreement with the results of luciferase activity and mRNA half-life results, the Cy3-labeled ADAM17 mRNA was significantly more localized to the FTO protein in the LPS-treated group compared with the untreated group, as detected by AlexaFluor488 fluorescence. We found that BV2 of oeFTO group possessed the strongest yellow signal with a significant positive correlation coefficient of $\mathrm{Rr}$ value both in scatter and line profile analyze responding to LPS (Fig. $5 \mathrm{a}$, b). Given that the silencing of FTO promoted the expression of ADAM17 in the polarization process of BV2 cells, we next inhibited ADAM17 expression to examine whether M1 polarization could be blocked. As we expected, despite LPS stimulation, inhibition of ADAM17 reduced the FTO-mediated M1 polarization in BV2 cells treated with TAPI-1. To further examine the ADAM17-mediated effects on M1 polarization, we detected a number of genes associated M1/M2 polarization (Fig. 5c, d) and tested a series of pro-inflammatory and antiinflammatory factors by ELISA (Fig. 5e). Taken together, we concluded that reduced ADAM17 expression resulted in increased FTO expression to promote M1 polarization of BV2 cells.

\section{FTO is closely related to brain injury after TBI}

Page $11 / 30$ 
A TBI model was used to verify whether FTO had an effect on microglial polarization during the initial inflammatory process caused by brain injury in vivo (Fig. 6a). Western blot results showed that FTO levels decreased significantly at days 1,3 , and after TBI, with the most significant decline on day $3(P<0.05)$, and gradually increased near baseline levels on day 14 after injury (Fig. 6b). Immunohistochemistry also showed that FTO in the cortical injury area was significantly reduced at day 3 after in the TBI group (Fig. $6 \mathrm{c})$. The results of immunofluorescence double staining showed that FTO was mainly expressed in microglia (Fig. 6d). Over-expression of FTO induced by NADP has been verified [33], and our results were in agreement Fig. $6 \mathrm{e}$.

Modified neurological severity scoring (mNSS), brain water, and Evans blue dye content were used to evaluate post-TBI neurological function after over-expression of FTO. The results showed that, compared with the TBI group, the mNSS of the TBI+NADP group was remarkably improved at day 3 after TBI (Fig. 6f). Compared with the TBI group, the brain water content and the penetration rate of Evans blue in the TBI+NADP group were significantly reduced compared with the TBI group (Fig. $6 g-i)$.

Concurrently, compared with the TBI group, the neuronal apoptosis rate in the TBI+NADP group was significantly lower at day 3 after TBI compared with the TBI group (Fig. 7a-c). The number of M1 microglia (CD86+/lba+) in the TBI+NADP group was lower, whereas the number of M2 microglia (CD206, Arg-1) was significantly increased, compared with that in the TBI group. (Fig. 7d, e). Finally, the production of proinflammatory cytokines decreased remarkably in the TBI+NADP group (Fig. 7f). These data demonstrate that over-expression of FTO inhibited microglia-induced inflammation and improved neurological function after TBI.

\section{FTO regulates neuroinflammation after TBI by targeting ADAM17 in microglia}

Western blot results showed that NADP intervention highly inhibited ADAM1 , TNF-alpha, and NF-KB p65 expression (Fig. 8a). Subsequently, exosomes in the supernatant of ADAM17-BV2 overexpression group were collected for identification, concentration, and quantification (Suppl. Fig. 1a-c). Based on the experimental results of the TBI group and TBI+NADP group (Fig. 6-7), we selected the intervention at 3 days after TBI (Fig. 8b, Schematic Diagram of Modeling). Nissl staining and Evans blue dye were used to evaluate the therapeutic effect of exo-oeADAM17 on neurological function after FTO overexpression. Compared with the TBI+NADP group, the apoptosis rate of neurons in the TBI+NADP+exo-oeADAM17 group was significantly increased (Fig. 8c). Meanwhile, the penetration of the corresponding Evans blue dye in the TBI+NADP+exo-oeADAM17 group was significantly increased (Fig. 8d). Compared with the TBI+NADP group, the TBI+NADP+exo-oeADAM17 group had a higher level of the M1 microglial biomarkers CD86 and iNOS (Fig. 8e), and the production of pro-inflammatory cytokines was remarkably increased (Fig. 8f). These data indicate that the over-expression of FTO contributed to the inhibition of the inflammation induced by microglia and improved the nerve function after brain trauma.

\section{Discussion}


The main findings of this study include: 1) In BV2 cells and mice with TBI, the expression of FTO in the LPS treatment group was significantly down-regulated. The down-regulation of FTO expression increased the $\mathrm{m}^{6} \mathrm{~A}$ level in $\mathrm{M} 1$ microglia in the entire transcriptome. 2) After FTO interference, the M1/M0 phenotype detection experiments revealed the BV2 cells shifted from the M0 to M1 the phenotype as the population

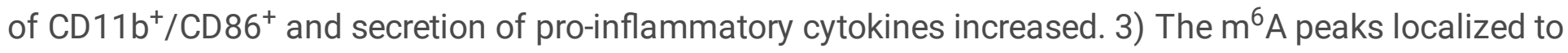
the ADAM17 and TNF-a genes increased, especially in the 3'UTR and 5'UTR regions of the ADAM17 gene. 4) FTO may affect the transcription modification of ADAM17 and the expression of downstream factors associated with the TNF-a/NF-kB pathway. 5) Inhibition of ADAM17 blocked the M1-phenotypic transformation of microglia caused by FTO-m ${ }^{6}$ A-modification. In short, our study found that FTO-related $\mathrm{m}^{6} \mathrm{~A}$ modification regulated the activation of microglia and neuroinflammatory response. FTO regulated the inflammatory response induced by microglia by stabilizing the expression of ADAM17, and may be considered as a new potential target for the treatment of brain injury (Fig. 9).

Our previous studies confirmed that the neuroinflammatory response, mediated by microglial polarization, plays an important role in the secondary nerve injury after $\operatorname{TBI}[3,8,9]$. Exploring the molecular mechanisms of microglial polarization regulation is particularly important for improving neurological function after TBI. The results of the present study revealed that $\mathrm{m}^{6} \mathrm{~A}$ methylation was related to microglial polarization. Here, we found that FTO down-regulated the translation and expression of ADAM17 gene by inhibiting the 3'UTR and 5'UTR $\mathrm{m}^{6} \mathrm{~A}$ modification levels of ADAM17 mRNA in microglia, which affected the expression of downstream factors associated with the TNF- $a / N F-k B$ pathway, thereby inhibiting M1 microglial polarization. Down-regulation of FTO led to abnormally high expression of ADAM17 in microglia, which promoted inflammation after TBI (Fig. 9).

$M^{6} \mathrm{~A}$ modification is one of the most common ways to modify mRNA in eukaryotic cells [21, 24]. As the first identified $\mathrm{m}^{6} \mathrm{~A}$ demethylase, FTO was found to be involved in the regulation of dopamine signal transduction in the midbrain of mice $[27,41]$. The midbrain includes areas associated with the formation of learning and memory, as well as adult neurogenesis [41]. FTO also has an important contribution to immune inflammation $[42,43]$. However, the role of FTO in TBI is not fully understood. More importantly, the role of FTO in the immunophenotypic transition of microglia remains unclear. This study has found that FTO regulates the expression of ADAM17 in microglia by inhibiting $\mathrm{m}^{6} \mathrm{~A}$ modification, which in turn blocked the immunophenotypic transformation of microglia, thereby affecting the early inflammatory response after TBI. Although we have revealed the epigenetic regulation of FTO in microglia, another $\mathrm{m}^{6} \mathrm{~A}$ methyltransferase, METTL3, was previously found to promote LPS-induced microglial inflammation by activating the TRAF6/NF-KB signal pathway $[24,44]$. METTL3 also utilizes $\mathrm{m}^{6} \mathrm{~A}$ to up-regulate the expression of TRAF6, and promote the expression of inflammatory cytokines and proteins related to the

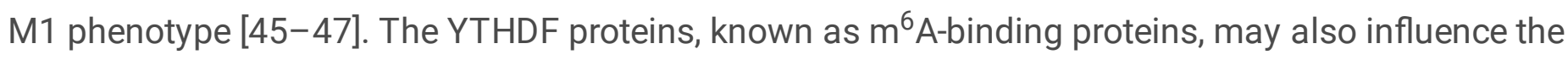
stability of methylated RNA to regulate transcription $[37,48]$. In this study, we systematically analyzed the key regulatory factors of $\mathrm{m}^{6} \mathrm{~A}$ modification that were related to $\mathrm{M} 1$ microglial polarization after TBI and in an LPS-induced microglial cell line. We found that the expression of YTHDF1 and YTHDF2 increased in 
the M1 phenotype group compared with that of the $\mathrm{M} 0$ phenotype group but that the difference was not statistically significant. FTO and METTL3 were decreased in the M1 phenotype group compared with the M0 phenotype group and, in particular, the FTO level decreased significantly. The down-regulation of FTO expression was closely related to the polarization of microglia after TBI. Compared with the M0 normal group, the overall level of $\mathrm{m}^{6} \mathrm{~A}$ in $\mathrm{M} 1$ microglia induced by LPS or after TBI was significantly increased. Further studies confirmed that the changes in methylation levels were related to the low expression of FTO and clarified the role of FTO in the regulation of microglial M1 polarization during the early stages of TBI.

ADAMs are a family of metalloendopeptidases belonging to the zinc dependent superfamily of enzymes, which are involved in a variety of biological processes [10]. ADAM protein sheddase activity mediates the separation of the extracellular domain of membrane-anchored receptors that can cleave a variety of substrates, including growth factors (all the EGFR ligands), cytokines (e.g., pro-TNF-a), cytokine receptors (e.g., IL-6R, TNF-R, and TGF- $\beta$ Rs), ErbB ligands (e.g., TGF- $\alpha$ and TGF- $\beta$ ), and amyloid precursor protein [11, $12,49,50]$. In particular, TNF-a, TGF-a, and amphiregulin are cleaved by ADAM17 sheddase activity [11, 12]. TNF-a is a type II transmembrane protein expressed on the cell surface in a membrane-bound form. After cleavage by ADAM17, soluble TNF-a binds to the TNF-a R to activate the NF-KB-related signaling pathway, which in turn initiates and regulates the inflammation cascade $[14,15]$. According to our data, TNF-a/NF-kB signaling pathway genes were enriched in LPS-stimulated BV2 cells (M1 phenotype) and that the $\mathrm{m}^{6} \mathrm{~A}$ motifs were enriched in ADAM17 and TNF-a mRNA transcripts, but not in ADAM10. Our data indicated that the ADAM17/TNF-a/NF-kB pathway genes are affected by FTO-mediated $\mathrm{m}^{6} \mathrm{~A}$ modification and are critical to M1 microglial polarization.

In order to confirm that ADAM17 is the main downstream target of FTO-mediated regulation of M1 microglial polarization, we screened differentially modified gene transcripts through $\mathrm{m}^{6} \mathrm{~A}$-sequencing. Through mRNA half-life and protein half-life experiments, the regulatory mechanism of FTO on ADAM17 was analyzed at both the molecular and protein levels. The results showed that FTO participates in the transcriptional regulation of ADAM17 by affecting the stability of ADAM17 mRNA and increasing the translational speed of ADAM17 protein. Double luciferase mutation further verified that siFTO mainly regulated the 3'UTR of ADAM17, suggesting that ADAM17 is the main downstream target of FTO. Finally, we confirmed that ADAM17 is the key gene target of FTO-mediated regulation of M1 microglial polarization, by showing that FTO overexpression specifically inhibited ADAM17 both in vitro and in vivo. Targeted overexpression of ADAM17 significantly counteracted the anti-inflammatory effects of M1 microglial polarization induced by FTO overexpression. In the future, research on FTO knockout mice should be carried out to study the mechanism of FTO-mediated inhibition of ADAM17 and regulation of microglial-induced inflammation.

\section{Conclusions}


In this study, we provide convincing evidence that FTO, as a key $\mathrm{m}^{6} \mathrm{~A}$ demethylase, is down-regulated in a TBI mouse model and that low expression of FTO induces an increase in the level of methylation that is significantly associated with microglial polarization. $\mathrm{M}^{6} \mathrm{~A}$ modification of FTO at the $3^{\prime} \mathrm{UTR}$ site of ADAM17 causes the degradation of ADAM17. In the TBI model, the low expression of FTO resulted in the up-regulation of ADAM17 expression and the M1 polarization of microglia M1, which was accelerated through epigenetic modification. In summary, our results indicate that FTO regulates the inflammatory response by inducing M1 microglial polarization and stabilizing the expression of ADAM17. FTO is expected to become a new target for the treatment of craniocerebral injury.

\section{Declarations}

\section{Ethics approval and consent to participate}

Human subjects or samples were not used in this study. All animal experiments were approved by the Ethics Committee of the Second Affiliated Hospital of Fujian Medical University.

\section{Consent for publication}

Consent for publication is not applicable for this manuscript.

\section{Availability of data and materials}

All the datasets and materials supporting the conclusions of this article are presented in the manuscript.

\section{Competing interests}

The authors declare that they have no competing interests.

\section{Funding}

This work was supported by grants from the funds for China Postdoctoral Science Foundation (2020M682069), Fujian Province Scientific Foundation (2019J01168), Young and middle-aged backbone talent foundation of Fujian Provincial Commission of Health Construction (2020GGA058) from Dr. Xiangrong Chen. We thank International Science Editing (http://www.internationalscienceediting.com ) for editing this manuscript.

\section{Authors' contributions}

X.C.,;conception and design, writing of the manuscript.

J.Y., Y.C., W.L. Y.L. and W.F.; supported several experiments, acquisition of data, analysis and interpretation of data. 
J.C., D.K., and C.D.; quality control and revision of the manuscript. All authors read and approved the final manuscript.

\section{Acknowledgments}

Not applicable.

\section{Author details}

a Department of Neurosurgery, Neurosurgery Research Institute, The First Affiliated Hospital of Fujian Medical University, Fuzhou, Fujian Province, China;

${ }^{b}$ Department of Neurosurgery, the Second Clinical Medical college, the Second Affiliated Hospital of Fujian Medical University, Quanzhou, Fujian Province, China;

c Department of Anesthesiology and Critical Care Medicine, Zhongshan Hospital, Fudan University, Shanghai, 200032, China

\section{References}

1. Shi K, Zhang J, Dong JF, Shi FD. Dissemination of brain inflammation in traumatic brain injury. Cell Mol Immunol. 2019;16:523-530.

2. Zhao Z, Zhou Y, Tian Y, Li M, Dong JF, Zhang J. Cellular microparticles and pathophysiology of traumatic brain injury. Protein Cell. 2017;8:801-810.

3. Wei G, Wang J, Wu Y, Zheng X, Zeng Y, Li Y, et al. Sirtuin 1 alleviates neuroinflammation-induced apoptosis after traumatic brain injury. J Cell Mol Med. 2021;25:4478-4486.

4. Scott MC, Bedi SS, Olson SD, Sears CM, Cox CS. Microglia as therapeutic targets after neurological injury: strategy for cell therapy. Expert Opin Ther Targets. 2021;25:365-380.

5. Li B, Dasgupta C, Huang L, Meng X, Zhang L. MiRNA-210 induces microglial activation and regulates microglia-mediated neuroinflammation in neonatal hypoxic-ischemic encephalopathy. Cell Mol Immunol. 2020;17:976-991.

6. Wen L, You W, Wang H, Meng Y, Feng J, Yang X. Polarization of Microglia to the M2 Phenotype in a Peroxisome Proliferator-Activated Receptor Gamma-Dependent Manner Attenuates Axonal Injury Induced by Traumatic Brain Injury in Mice. J Neurotrauma. 2018;35:2330-2340.

7. Hellström Erkenstam N, Smith PL, Fleiss B, Nair S, Svedin P, Wang W, et al. Temporal Characterization of Microglia/Macrophage Phenotypes in a Mouse Model of Neonatal Hypoxic-Ischemic Brain Injury. Front Cell Neurosci. 2016;10:286.

8. Chen X, Chen C, Fan S, Wu S, Yang F, Fang Z, et al. Omega-3 polyunsaturated fatty acid attenuates the inflammatory response by modulating microglia polarization through SIRT1-mediated deacetylation of the HMGB1/NF-kappaB pathway following experimental traumatic brain injury. $J$ Neuroinflammation. 2018;15:116. 
9. Chen X, Wu S, Chen C, Xie B, Fang Z, Hu W, et al. Omega-3 polyunsaturated fatty acid supplementation attenuates microglial-induced inflammation by inhibiting the HMGB1/TLR4/NF-KB pathway following experimental traumatic brain injury. J Neuroinflammation. 2017;14:143.

10. Hsia HE, Tüshaus J, Brummer T, Zheng Y, Scilabra SD, Lichtenthaler SF. Functions of 'A disintegrin and metalloproteases (ADAMs)' in the mammalian nervous system. Cell Mol Life Sci. 2019;76:30553081.

11. Schumacher N, Rose-John S, Schmidt-Arras D. ADAM-Mediated Signalling Pathways in Gastrointestinal Cancer Formation. Int J Mol Sci. 2020;21:5133.

12. Camodeca C, Cuffaro D, Nuti E, Rossello A. ADAM Metalloproteinases as Potential Drug Targets. Curr Med Chem. 2019;26:2661-2689.

13. Geribaldi-Doldán N, Carrasco M, Murillo-Carretero M, Domínguez-García S, García-Cózar FJ, MuñozMiranda JP, et al. Specific inhibition of ADAM17/TACE promotes neurogenesis in the injured motor cortex. Cell Death Dis. 2018;9:862.

14. Bell JH, Herrera AH, Li Y, Walcheck B. Role of ADAM17 in the ectodomain shedding of TNF-alpha and its receptors by neutrophils and macrophages. J Leukoc Biol. 2007;82:173-176.

15. Qing X, Chinenov Y, Redecha P, Madaio M, Roelofs JJ, Farber G, et al. iRhom2 promotes lupus nephritis through TNF-a and EGFR signaling. J Clin Invest. 2018;128:1397-1412.

16. Yu H, Lin L, Zhang Z, Zhang H, Hu H. Targeting NF-kB pathway for the therapy of diseases: mechanism and clinical study. Signal Transduct Target Ther. 2020;5:209.

17. Qu F, Tsegay PS, Liu Y. N(6)-Methyladenosine, DNA Repair, and Genome Stability. Front Mol Biosci. 2021;8:645823.

18. Coker H, Wei G, Brockdorff N. m6A modification of non-coding RNA and the control of mammalian gene expression. Biochim Biophys Acta Gene Regul Mech. 2019;1862:310-318.

19. Jiang X, Liu B, Nie Z, Duan L, Xiong Q, Jin Z, et al. The role of m6A modification in the biological functions and diseases. Signal Transduct Target Ther. 2021;6:74.

20. Zhang SY, Zhang SW, Liu L, Meng J, Huang Y. m6A-Driver: Identifying Context-Specific mRNA m6A Methylation-Driven Gene Interaction Networks. PLoS Comput Biol. 2016;12:e1005287.

21. Zhu ZM, Huo FC, Pei DS. Function and evolution of RNA N6-methyladenosine modification. Int J Biol Sci. 2020;16:1929-1940.

22. Song Y, Xu Q, Wei Z, Zhen D, Su J, Chen K, et al. Predict Epitranscriptome Targets and Regulatory Functions of N (6)-Methyladenosine (m(6)A) Writers and Erasers. Evol Bioinform Online. 2019;15:1176934319871290.

23. Huang J, Yin P. Structural Insights into N(6)-methyladenosine (m(6)A) Modification in the Transcriptome. Genomics Proteomics Bioinformatics. 2018;16:85-98.

24. Paramasivam A, Vijayashree Priyadharsini J. Novel insights into m6A modification in circular RNA and implications for immunity. Cell Mol Immunol. 2020;17:668-669. 
25. Paramasivam A, Priyadharsini JV, Raghunandhakumar S. Implications of m6A modification in autoimmune disorders. Cell Mol Immunol. 2020;17:550-551.

26. Hu Y, Feng Y, Zhang L, Jia Y, Cai D, Qian SB, et al. GR-mediated FTO transactivation induces lipid accumulation in hepatocytes via demethylation of m(6)A on lipogenic mRNAs. RNA Biol. 2020;17:930-942.

27. Bai L, Tang Q, Zou Z, Meng P, Tu B, Xia Y, et al. m6A Demethylase FTO Regulates Dopaminergic Neurotransmission Deficits Caused by Arsenite. Toxicol Sci. 2018;165:431-446.

28. Li Z, Weng H, Su R, Weng X, Zuo Z, Li C, et al. FTO Plays an Oncogenic Role in Acute Myeloid Leukemia as a N(6)-Methyladenosine RNA Demethylase. Cancer Cell. 2017;31:127-141.

29. Bartosovic M, Molares HC, Gregorova P, Hrossova D, Kudla G, Vanacova S. N6-methyladenosine demethylase FTO targets pre-mRNAs and regulates alternative splicing and 3'-end processing. Nucleic Acids Res. 2017;45:11356-11370.

30. Yu J, Zhang Y, Ma H, Zeng R, Liu R, Wang P, et al. Epitranscriptomic profiling of N6-methyladenosinerelated RNA methylation in rat cerebral cortex following traumatic brain injury. Mol Brain. 2020;13:11.

31. Kim Y, Fu AH, Tucker LB, Liu J, McCabe JT. Characterization of controlled cortical impact devices by high-speed image analysis. J Neurosci Res. 2018;96:501-511.

32. Siebold L, Obenaus A, Goyal R. Criteria to define mild, moderate, and severe traumatic brain injury in the mouse controlled cortical impact model. Exp Neurol. 2018;310:48-57.

33. Wang L, Song C, Wang N, Li S, Liu Q, Sun Z, et al. NADP modulates RNA m(6)A methylation and adipogenesis via enhancing FTO activity. Nat Chem Biol. 2020;16:1394-1402.

34. Zheng L, Tang X, Lu M, Sun S, Xie S, Cai J, et al. microRNA-421-3p prevents inflammatory response in cerebral ischemia/reperfusion injury through targeting m6A Reader YTHDF1 to inhibit p65 mRNA translation. Int Immunopharmacol. 2020;88:106937.

35. Zan J, Zhang H, Gu AP, Zhong KL, Lu MY, Bai XX, et al. Yin Yang 1 Dynamically Regulates Antiviral Innate Immune Responses During Viral Infection. Cell Physiol Biochem. 2017;44:607-617.

36. Meng J, Lu Z, Liu H, Zhang L, Zhang S, Chen Y, et al. A protocol for RNA methylation differential analysis with MeRIP-Seq data and exomePeak R/Bioconductor package. Methods. 2014;69:274-281.

37. Chen YG, Chen R, Ahmad S, Verma R, Kasturi SP, Amaya L, et al. N6-Methyladenosine Modification Controls Circular RNA Immunity. Mol Cell. 2019;76:96-109.e109.

38. Vargas AM, Rivera-Rodriguez DE, Martinez LR. Methamphetamine alters the TLR4 signaling pathway, NF-KB activation, and pro-inflammatory cytokine production in LPS-challenged NR-9460 microglialike cells. Mol Immunol. 2020;121:159-166.

39. Arenas YM, Cabrera-Pastor A, Juciute N, Mora-Navarro E, Felipo V. Blocking glycine receptors reduces neuroinflammation and restores neurotransmission in cerebellum through ADAM17-TNFR1-NF-K $\beta$ pathway. J Neuroinflammation. 2020;17:269.

40. Zunke F, Rose-John S. The shedding protease ADAM17: Physiology and pathophysiology. Biochim Biophys Acta Mol Cell Res. 2017;1864:2059-2070. 
41. Li L, Zang L, Zhang F, Chen J, Shen H, Shu L, et al. Fat mass and obesity-associated (FTO) protein regulates adult neurogenesis. Hum Mol Genet. 2017;26:2398-2411.

42. Hu F, Tong J, Deng B, Zheng J, Lu C. MiR-495 regulates macrophage M1/M2 polarization and insulin resistance in high-fat diet-fed mice via targeting FTO. Pflugers Arch. 2019;471:1529-1537.

43. Zhou Y, Hambly BD, McLachlan CS. FTO associations with obesity and telomere length. J Biomed Sci. 2017;24:65.

44. Feng Z, Li Q, Meng R, Yi B, Xu Q. METTL3 regulates alternative splicing of MyD88 upon the lipopolysaccharide-induced inflammatory response in human dental pulp cells. J Cell Mol Med. 2018;22:2558-2568.

45. Wen L, Sun W, Xia D, Wang Y, Li J, Yang S. The m6A methyltransferase METTL3 promotes LPSinduced microglia inflammation through TRAF6/NF-kappaB pathway. Neuroreport. 2020; doi: 10.1097/WNR.0000000000001550.

46. Li D, Cai L, Meng R, Feng Z, Xu Q. METTL3 Modulates Osteoclast Differentiation and Function by Controlling RNA Stability and Nuclear Export. Int J Mol Sci. 2020;21:1660.

47. Zong X, Zhao J, Wang H, Lu Z, Wang F, Du H, et al. Mettl3 Deficiency Sustains Long-Chain Fatty Acid Absorption through Suppressing Traf6-Dependent Inflammation Response. J Immunol. 2019;202:567-578.

48. Tsuchiya $K$, Yoshimura K, Inoue Y, Iwashita Y, Yamada H, Kawase A, et al. YTHDF1 and YTHDF2 are associated with better patient survival and an inflamed tumor-immune microenvironment in nonsmall-cell lung cancer. Oncoimmunology. 2021;10:1962656.

49. Menghini R, Fiorentino L, Casagrande V, Lauro R, Federici M. The role of ADAM17 in metabolic inflammation. Atherosclerosis. 2013;228:12-17.

50. Düsterhöft S, Lokau J, Garbers C. The metalloprotease ADAM17 in inflammation and cancer. Pathol Res Pract. 2019;215:152410.

\section{Figures}



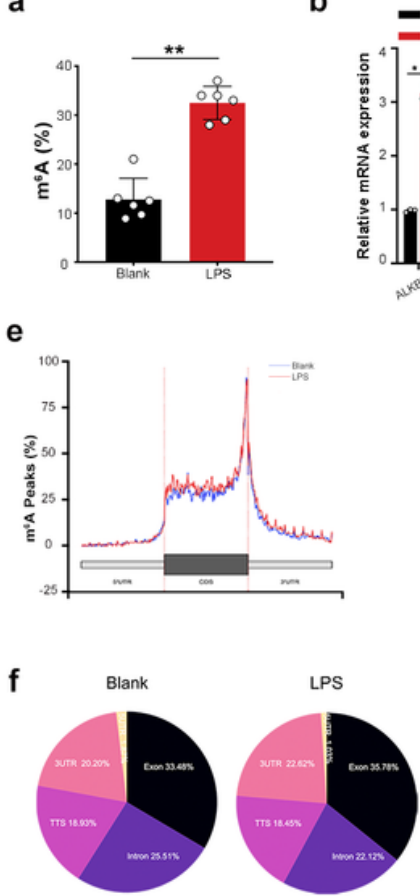

b

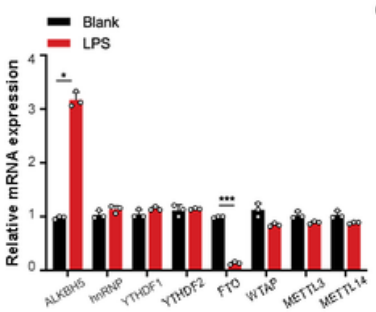

g
C
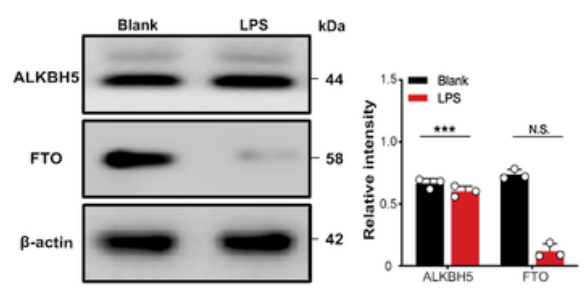

d

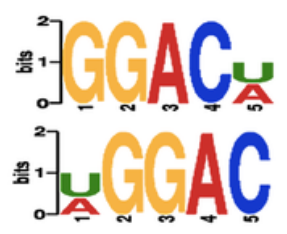

h

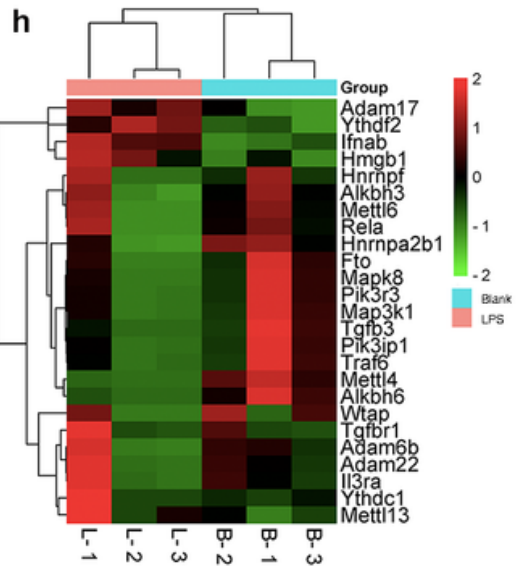

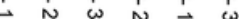

Figure 1

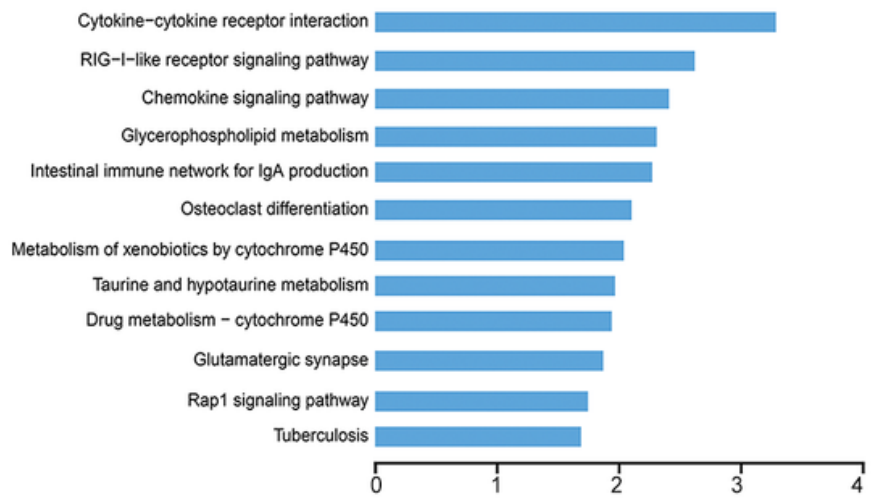

The level of $\mathrm{m}^{6} \mathrm{~A}$ modification increases when $\mathrm{M} 1$ microglia are activated. a $\mathrm{M}^{6} \mathrm{~A}$ RNA methylation quantitative experiments showed that compared with Blank (M0 phenotype) BV2 cells, the $\mathrm{m}^{6} \mathrm{~A}$ modification level of LPS-treated (M1 phenotype) BV2 cells was significantly increased $(P<0.05)$. $\mathbf{b}$ The expressions of hnRNP, YTHDF1, and YTHDF2 increased in the M1 phenotype group compared with the M0 phenotype group, although the difference was not statistically significant. By contrast, FTO, WTAP, METTL3, and METTL14 decreased in the M1 phenotype group, with the FTO level showing a significant reduction $(P<0.05)$. $c$ Western blot also indicated a lower expression of FTO in the M1 phenotype group $(P<0.05)$ but with no significant change in the expression of ALKBH5. d Motif enrichment analysis revealed that the $\mathrm{m}^{6} \mathrm{~A}$ peaks identified above shared a common sequence element [U]GGAC[U]A. The consensus motif GGAC was detected both in M0 and M1 BV2 cells. e The density distribution pattern of $\mathrm{m}^{6} \mathrm{~A}$ peaks across the length of transcripts. Regions of the $5^{\prime} \mathrm{UTR}, \mathrm{CDS}$, and $\mathrm{3}^{\prime} \mathrm{UTR}$ were binned into 30 , 40 , and 30 segments according to their relative lengths, respectively, and the percentage of $\mathrm{m}^{6} \mathrm{~A}$ peaks fell within each region. $f$ The $\mathrm{m}^{6} \mathrm{~A}$ peak distribution in the $5^{\prime} \mathrm{UTR}$, start codon, CDS, stop codon, and $3^{\prime} \mathrm{UTR}$ across the entire set of mRNA transcripts. $\mathbf{g}$ GO enrichment analysis of genes transcripts with more than a two-fold expression in LPS-stimulated BV2 cells compared with control cells in the biological process category. $\mathbf{h}$ Heat map of differentially expressed genes in control and LPS-stimulated BV2 cells. Values are expressed as mean \pm SD from at least 3 independent experiments and the dots represent the value of each experiment. N.S. , nonsignificant, $P>0.05 ; *, P<0.05 ; * \star, P<0.01 ; * \star \star, P<0.001$. 


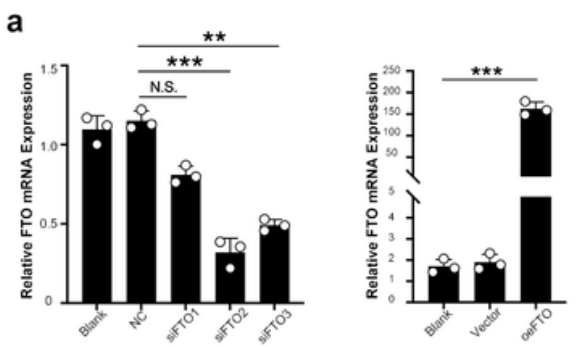

C
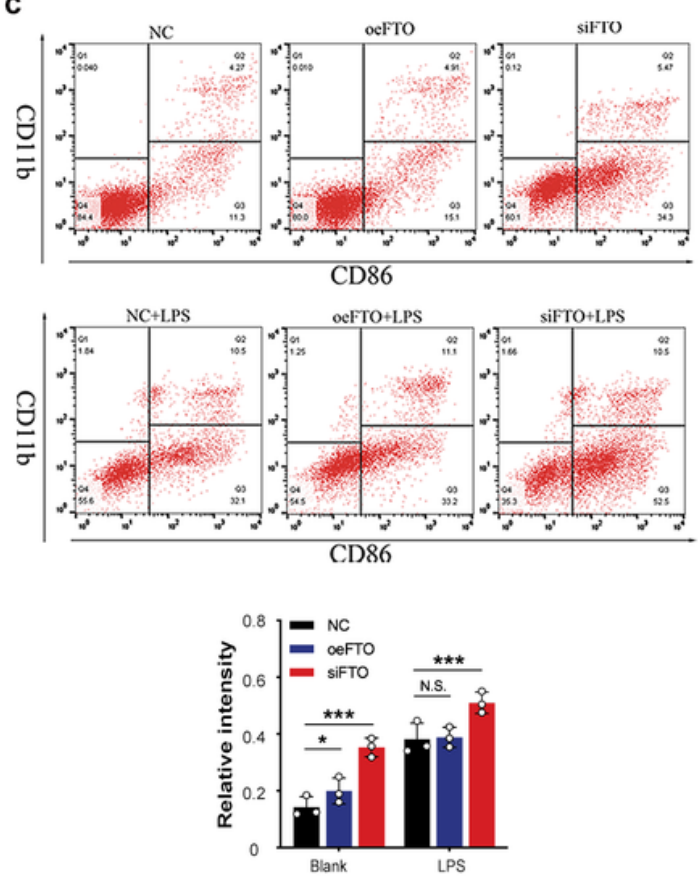

b

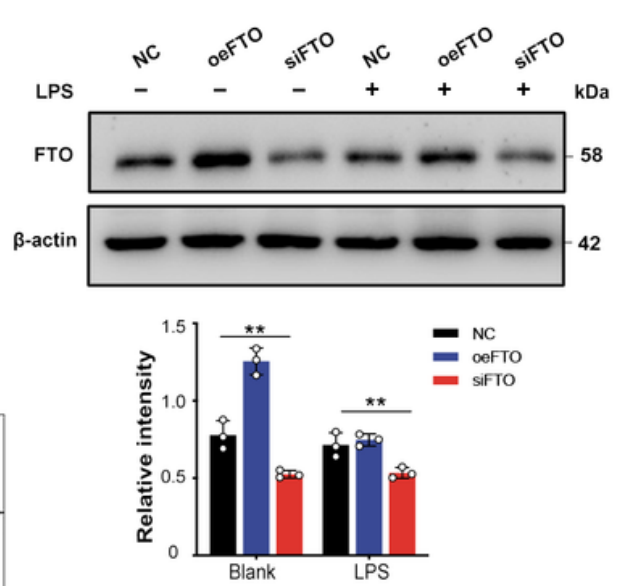

f

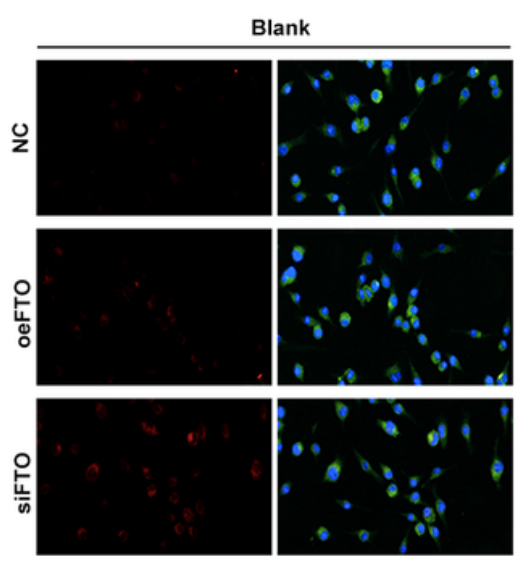

d
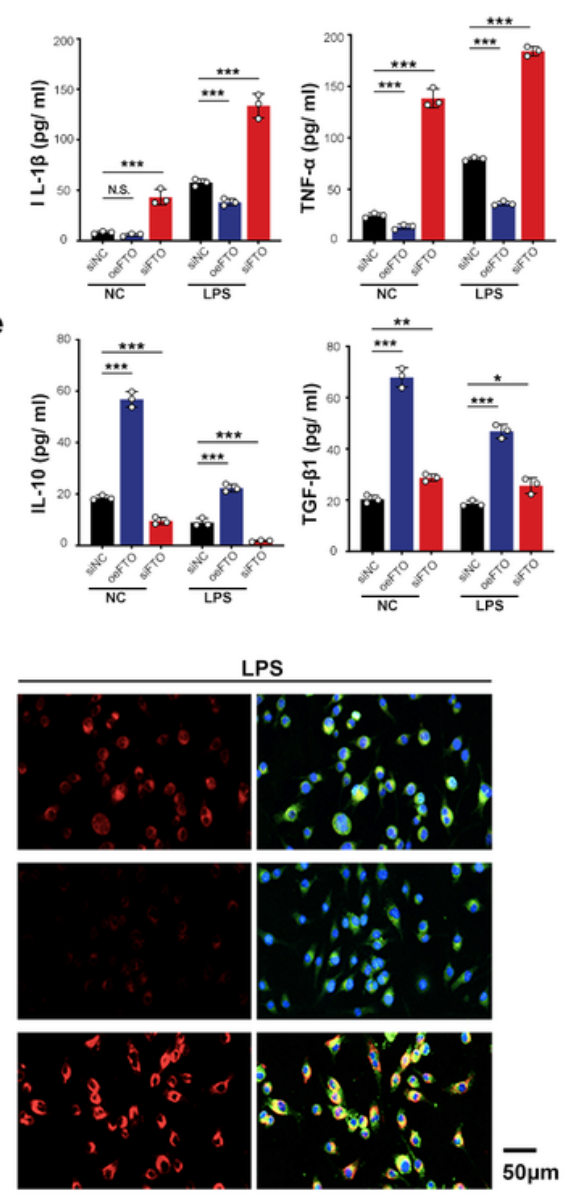

Figure 2

The effects of FTO on the microglial polarization. a,b The transfection efficiency of FTO knockdown and over-expression vectors was measured by qRT-PCR (a) and western blotting (b) in BV2 cells. c Expression of M1 biomarkers in FTO overexpression and siFTO BV2 cells in response to LPS-stimulation, as detected by flow cytometry. The siFTO group had a higher rate of $M 1$ phenotype $\left(C D 11 b^{+} / C D 86^{+}\right)$microglia in response to LPS stimulation $(P<0.05)$, compared with the negative control and FTO overexpression groups. $\mathbf{d}$ The production of proinflammatory cytokines (IL-1 $\beta$ and TNF- $\alpha$ ) increased remarkably in the siFTO group with LPS stimulation and decreased in FTO overexpression group with LPS stimulation. $\mathbf{e}$ The production of anti-inflammatory cytokines (IL-10 and TGF- 31 ) decreased remarkably in the siFTO group with LPS stimulation. $\mathrm{f}$ Immunofluorescence staining of CD 86 and Iba-1 in the FTO overexpression, siFTO, and negative control groups with or without LPS treatment. The siFTO group had a higher rate of M1 phenotype (CD86+//ba- $\left.1^{+}\right)$microglia in response to LPS stimulation compared with the negative control and FTO groups $(P<0.05)$. Representative photomicrographs of CD86-positive microglia are shown. Scale bars $=50 \mu \mathrm{m}$. Values are expressed as mean \pm SD from at least 3 independent experiments and the dots represent the value of each experiment. N.S. , nonsignificant, $P>0.05 ; *, P<0.05 ; \star \star, P<$ $0.01 ; * \star *, P<0.001$. 
a

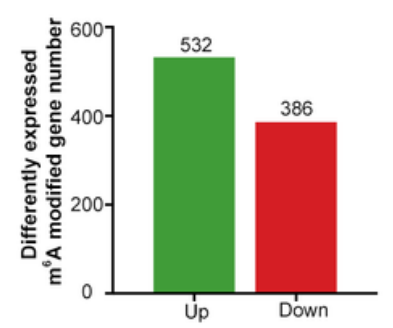

b

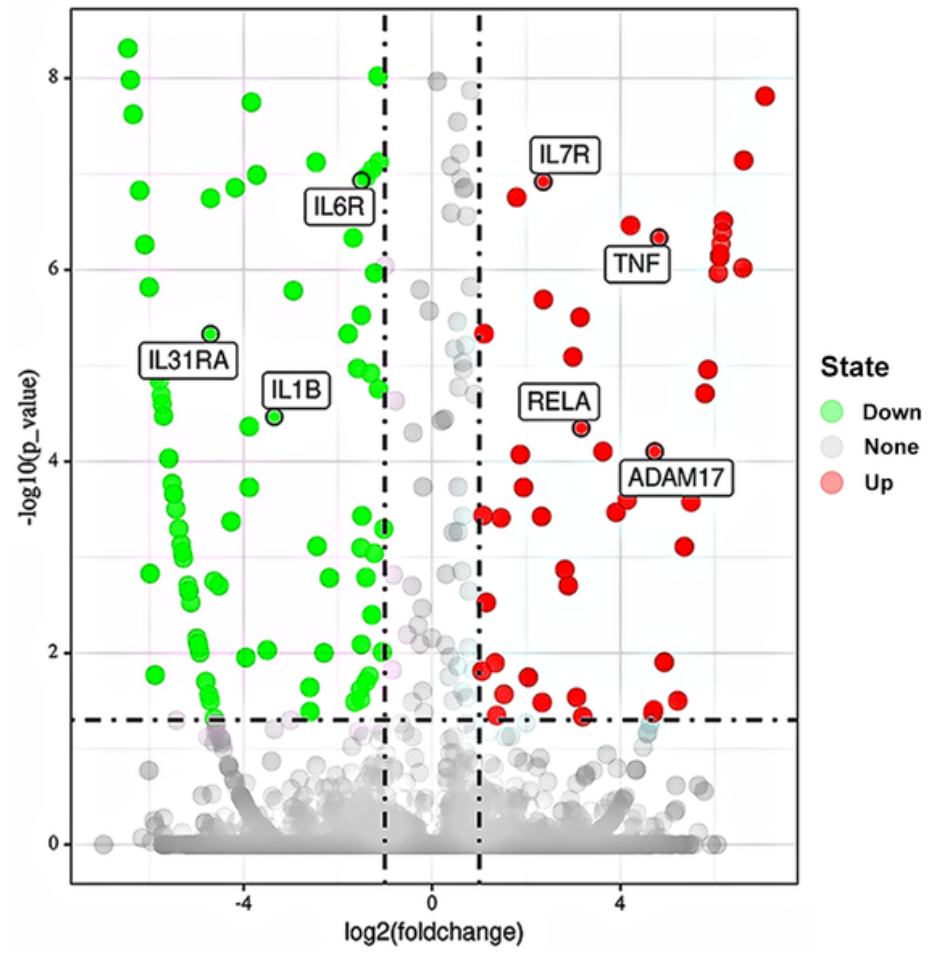

d

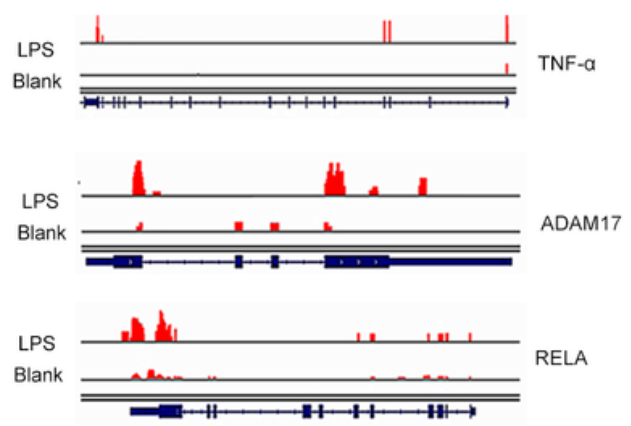

e

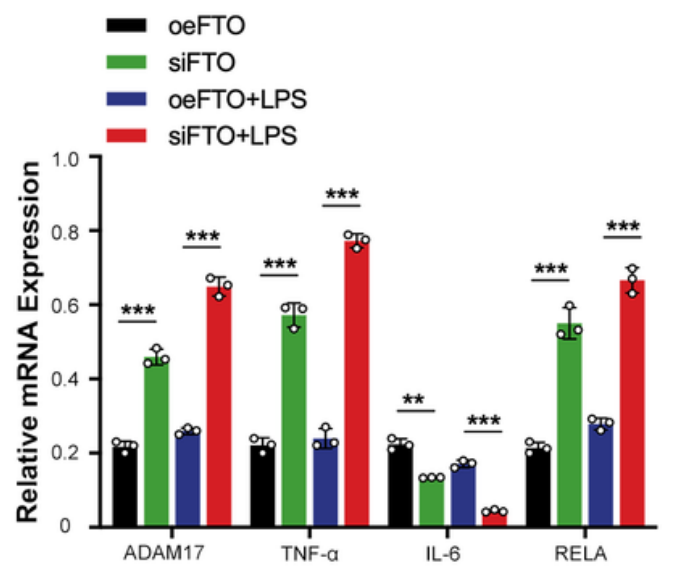

f
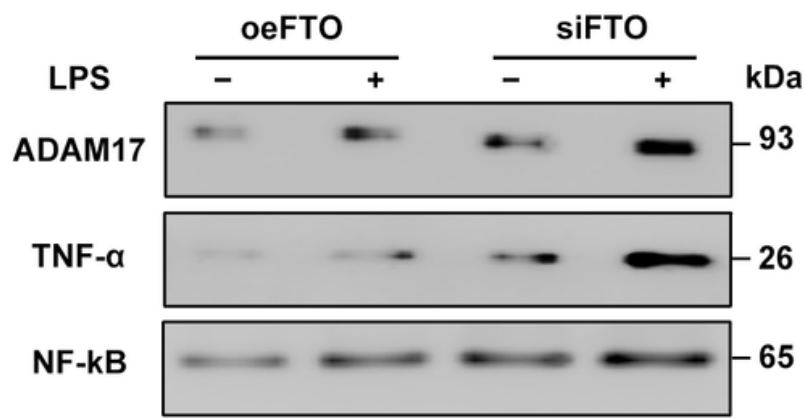

$\beta$-actin
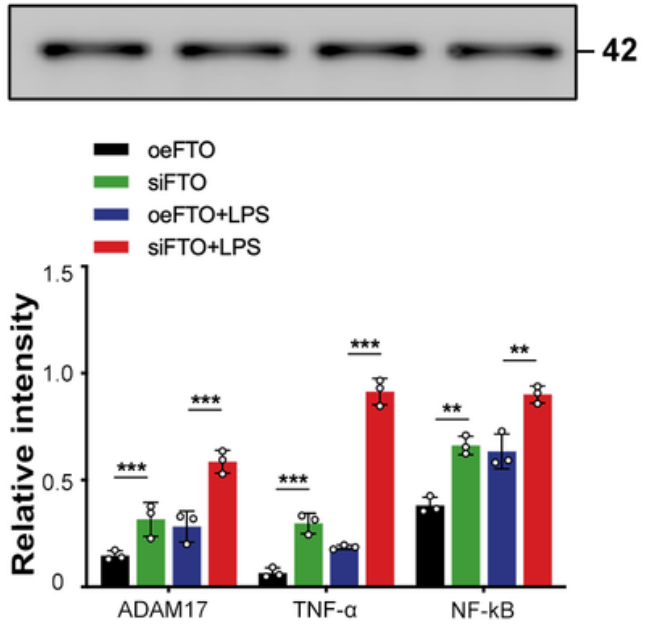

Figure 3

ADAM17 was a downstream target of FTO-mediated $\mathrm{m}^{6} \mathrm{~A}$ modification in microglia. a The $\mathrm{m}^{6} \mathrm{~A}$ modified genes were significantly different between blank and LPS-treated BV2 cells. $\mathbf{b}$ The overlap between $\mathrm{m}^{6} \mathrm{~A}$ modified genes and inflammatory response-related genes was analyzed in blank and LPS-treated BV2 cells using Venn diagrams. c Volcano plot of the gene enrichment pathway in blank and LPS-treated BV2 cells. Compared with the FTO overexpression group, deficiency of FTO in BV2 cells caused an upregulation of the downstream effector molecules of the ADAM17/TNF- $a / N F-k B$ pathway, including 
ADAM17, NF-kB p65, IL-6, IL-1 $\beta$, and TNF- $a(P<0.05)$. $\mathbf{d}$ m6 A RNA-sequencing profile of blank and LPStreated BV2 cells. By comparing $\mathrm{m}^{6} \mathrm{~A}$-sequencing with FTO overexpression BV2 cells, ADAM17, TNF-a and NF-KB mRNA in siFTO cells was mainly concentrated in the CDS and 3'UTR regions. e, $f$ Compared with siFTO BV2 cells, the levels of ADAM17, TNF- $a$, and Nf-KB mRNA in FTO overexpression BV2 cells decreased (e), as did the protein levels $(\mathbf{f})$. Values are expressed as mean \pm SD from at least 3 independent experiments and the dots represent the value of each experiment. N.S. , nonsignificant, $P>$ $0.05 ; *, P<0.05 ; * \star, P<0.01 ; * \star \star, P<0.001$.

a

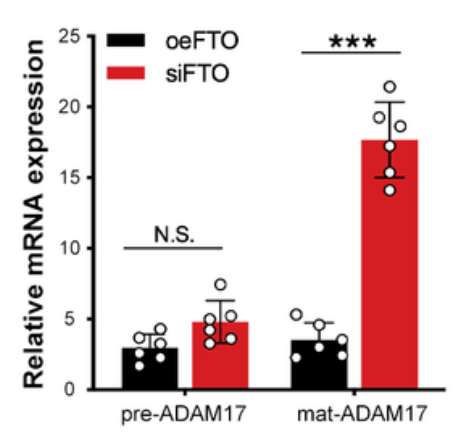

b

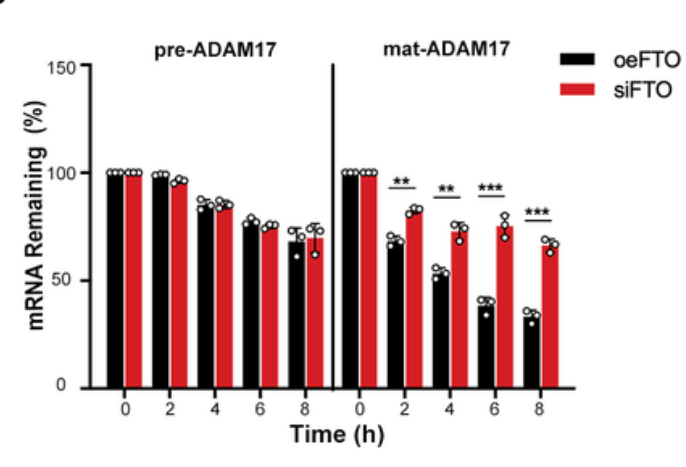

d

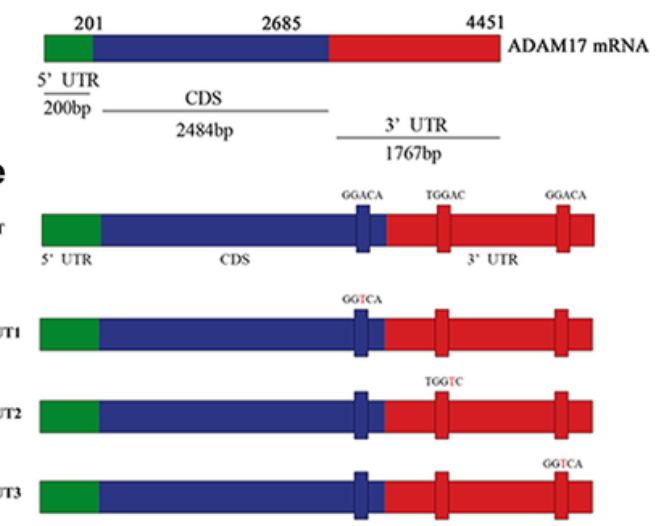

C

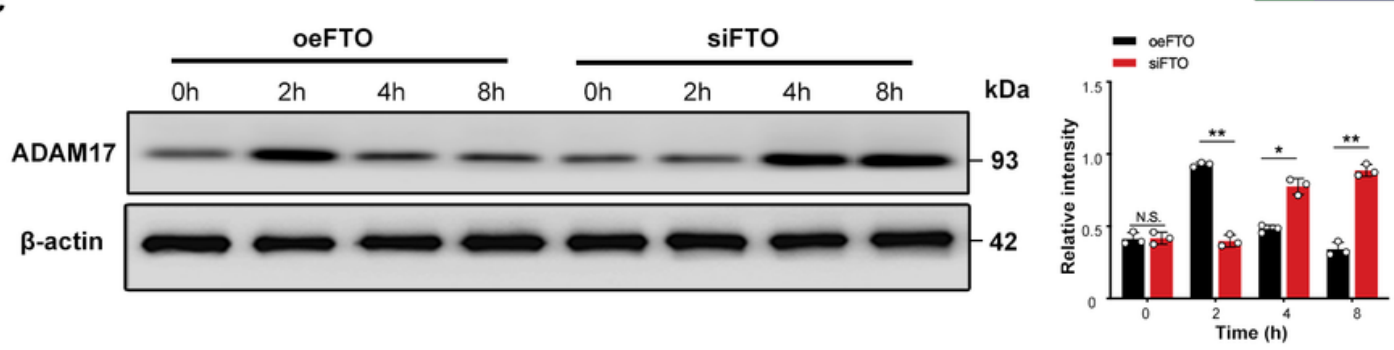

f

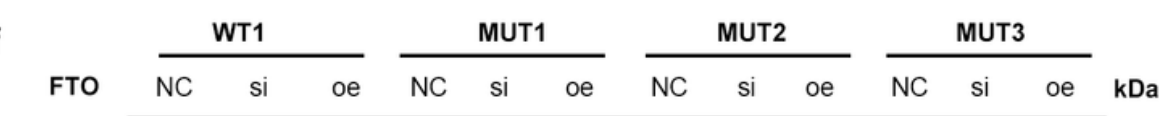
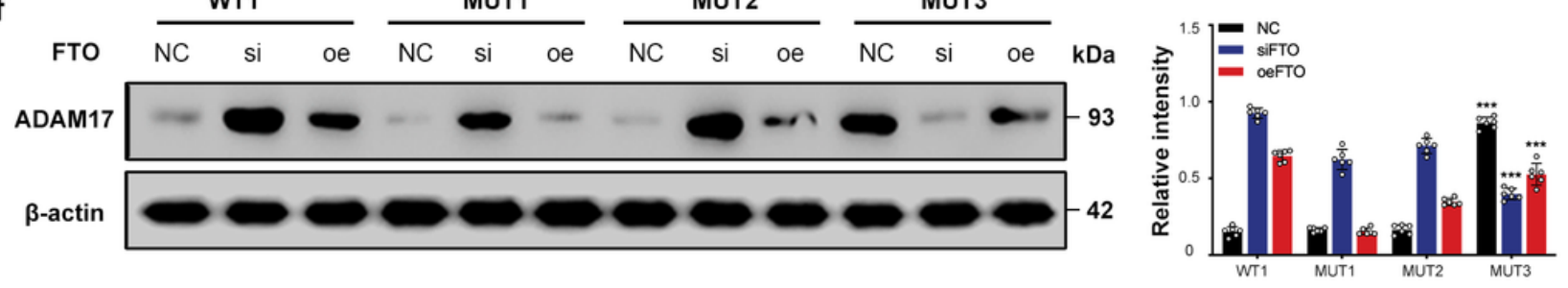

Figure 4

The epigenetic regulation of ADAM17 was by an FTO-m ${ }^{6}$ A-dependent mechanism. a Detection of ADAM17 precursor and mature transcripts by qPCR. Compared with FTO overexpression BV2 cells, the precursor mRNA and mature mRNA of ADAM17 in siFTO BV2 cells were significantly enhanced. $\mathbf{b}$ Detection of the half-life of ADAM17 in FTO overexpression and siFTO BV2 cells pretreated with actinomycin-D for 90 min and analyzed for precursor or mature ADAM17 mRNA at 0, 2, 4, 6, and $8 \mathrm{~h}$. The results revealed that in FTO overexpression and siFTO BV2 cells, precursor ADAM17 mRNA showed no significant difference, however, compared with the FTO overexpression BV2 cells, mature ADAM17 mRNA 
in the siFTO BV2 cells was significantly increased $(P<0.05)$. c The FTO overexpression and siFTO BV2 cells were pretreated with $\mathrm{CHX}$ for 90 min, and western blot analysis was applied to examine the expression of ADAM17. The results showed that compared with the cells treated with CHX, ADAM17 protein in the siFTO BV2 cells had a longer half-life $(P<0.05)$. $\mathbf{d}$ Schematic representation of positions of the $\mathrm{m}^{6} \mathrm{~A}$ motifs within ADAM17 mRNA. e Schematic representation of mutated (GGAC to GGTC) 3'UTR pmirGLO vector to investigate the role of $\mathrm{m}^{6} \mathrm{~A}$ in ADAM17 expression. $\mathbf{f} \mathrm{BV} 2$ cells transfected with pmirGLO-3'UTR or pmirGLO-3' UTR-Mut1/2 reporter plasmids. The mutant 03 of ADAM17 had markedly decreased luciferase activity compared with wild type BV2 cells, normalized to the Renilla data. Values are expressed as mean \pm SD from at least 3 independent experiments and the dots represent the value of each experiment. N.S. , nonsignificant, $P>0.05 ; *, P<0.05 ; * \star, P<0.01 ; * \star \star, P<0.001$.

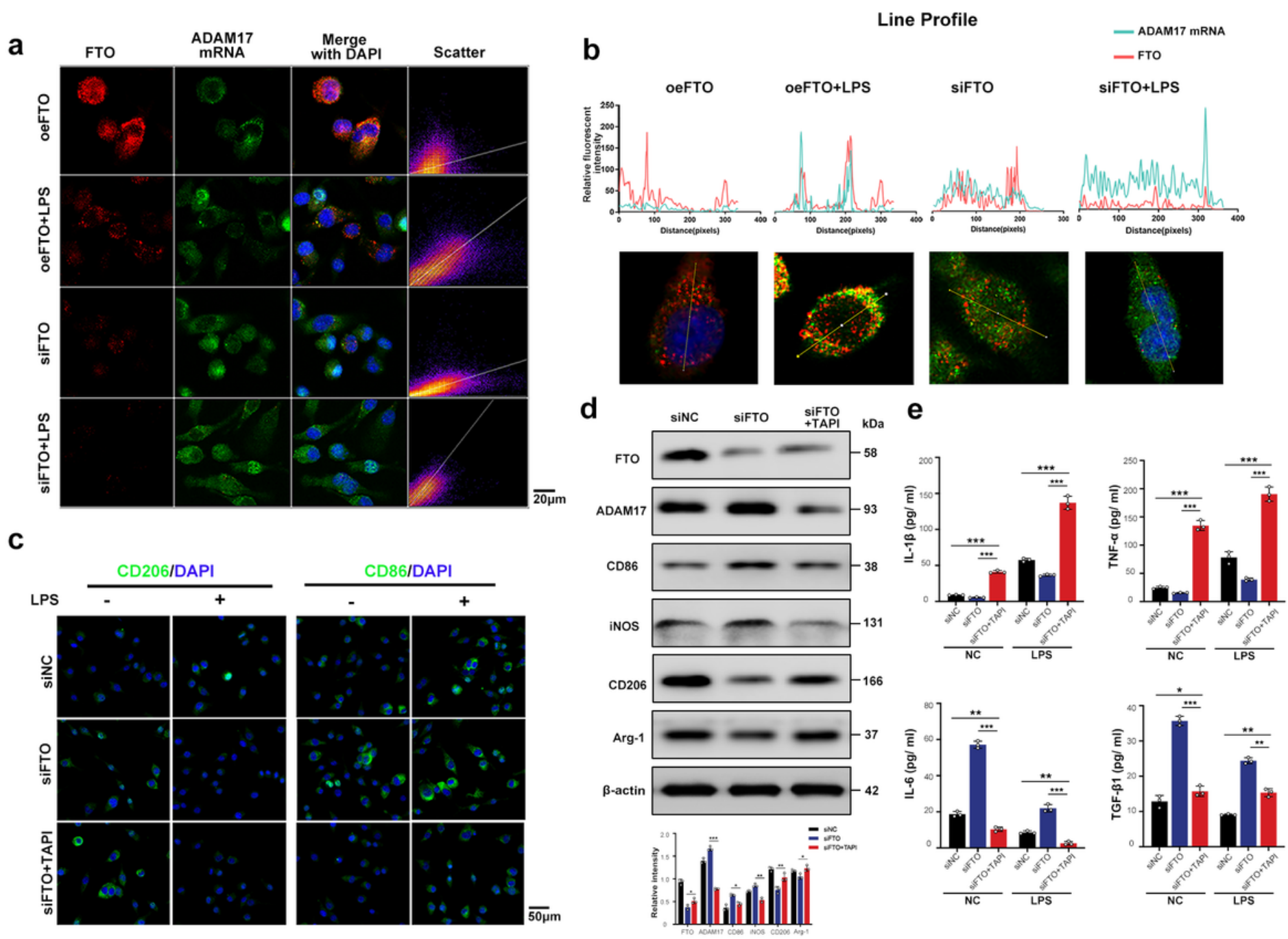

Figure 5

Inhibition of ADAM17 in vitro can block microglial polarization after FTO-m ${ }^{6} \mathrm{~A}$ modification. $\mathrm{a}, \mathrm{b}$ In the FTO overexpression (oeFTO) and FTO knockdown (siFTO) BV2 cells treated with or without LPS, in situ 
hybridization results indicated that ADAM17 co-localized with FTO (a). Scale bars $=20 \mu \mathrm{m}$. We found that BV2 cells of oeFTO group possessed the strongest yellow signal with a significant positive correlation coefficient of $\mathrm{Rr}$ value both in scatter and line profile analyze responding to LPS (b). c Suppression of ADAM17 alleviated reduced M1 (CD $\left.86^{+} / \mathrm{lba}-1^{+}\right)$microglial polarization in TAPI- 1 treated BV2 cells mediated by FTO, despite LPS treatment. Scale bars $=50 \mu \mathrm{m}$. d M1 phenotype biomarkers expression in the NC, siFTO, and siFTO+TAPI-1 groups. Compared with the siFTO group, the siFTO+TAPI-1 group had significantly reduced expression of CD86, ADAM17, and iNOS, and increased the expression of CD206 and Arg-1 $(P<0.05)$. e ELISA results showed that the siFTO+TAPI-1 group had significantly reduced expression of IL-1 $\beta$, TNF- $\alpha$, and IL- 6 and increased expression of TGF- $\beta 1$, compared with the siFTO group $(P<0.05)$. Values are expressed as mean \pm SD from at least 3 independent experiments and the dots represent the value of each experiment. N.S. , nonsignificant, $P>0.05 ;{ }^{*}, P<0.05$; $\star \star, P<0.01$; $* \star \star$, $P<0.001$.

a

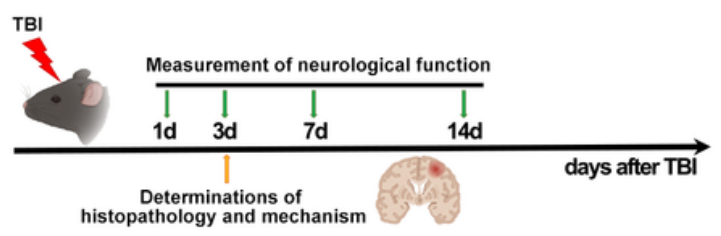

b
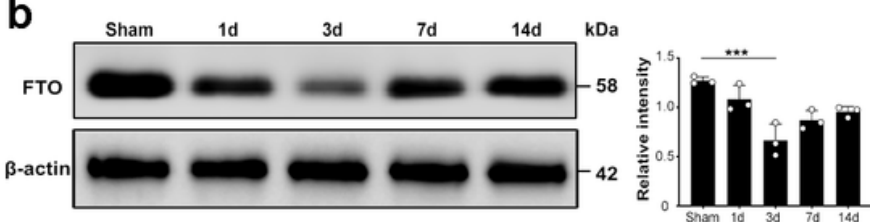

e

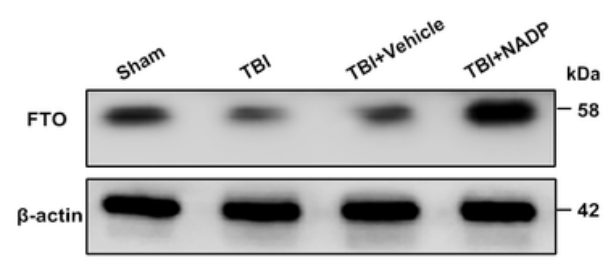

g
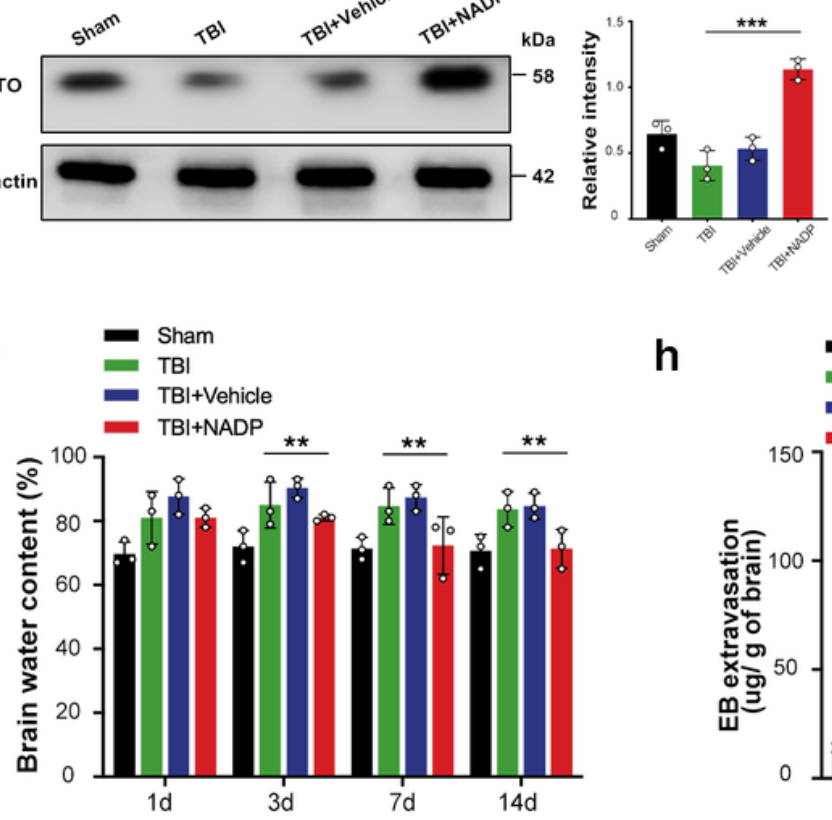

h
C
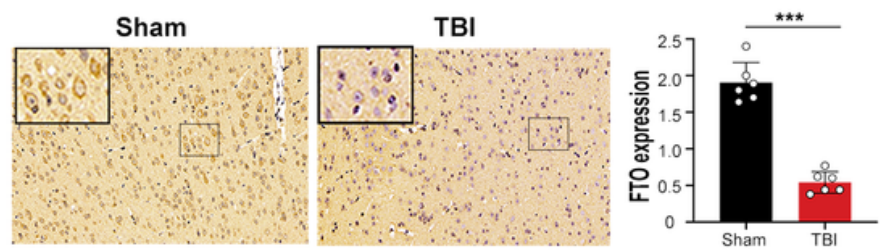

d
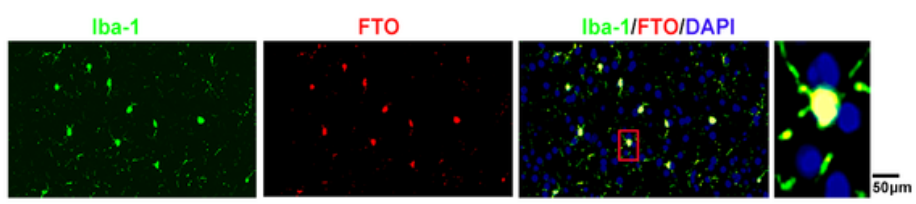

f

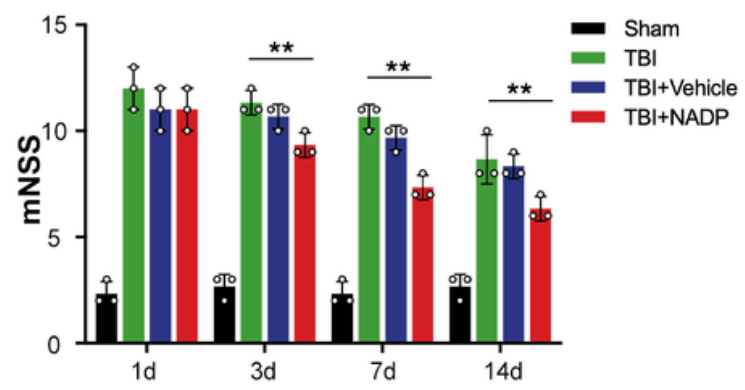

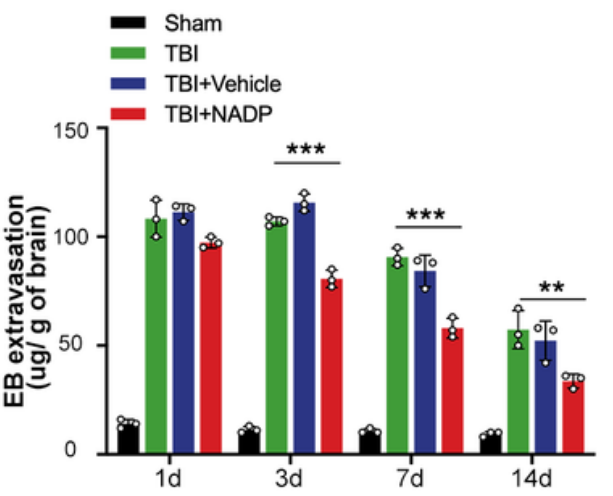

i
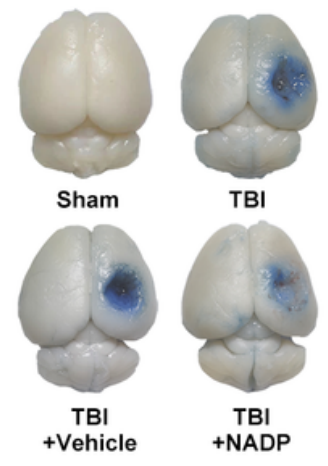

Figure 6 
FTO is closely related to brain injury after TBI. a Experimental scheme and a schematic brain section after TBI. The molecular biology study was performed on post-injury days $1,3,7$, and 14 . Areas in red refer to lesion sites. b The FTO level decreased significantly at days 1, 3 and 7 after TBI. In particular, the FTO level dropped most significantly at day 3 after TBI, after which the FTO level increased slowly to a near normal level at day 14 after TBI $(P<0.05)$. c Immunohistochemistry also showed that FTO expression in the cortical injury area was significantly reduced at day 3 after TBI the TBI group. Scale bars $=50 \mu \mathrm{m}$. $\mathbf{d}$ Double immunofluorescence staining showed that FTO was mainly expressed in microglia (Iba-1+). Representative photomicrographs of immunofluorescence double staining are shown. e Over-expression of FTO was induced by NADP. Compared with the TBI group, NADP intervention significantly increased FTO expression. $f$ Compared with the TBI group, the nerve function score of the TBI+NADP group was significantly improved at day 3 after TBI $(P<0.05)$. $\mathbf{g}$ The water content of brain tissue in the TBI+NADP group was significantly reduced at day 3 after TBI $(P<0.05) . \mathbf{h}$ The TBI+NADP group had significantly less extravasation of Evans Blue dye than the TBI group $(P<0.05)$. $\mathbf{i}$ Representative photos of Evans blue dye extravasation in the experimental groups at 3 days after the TBI. Values are expressed as mean \pm SD from at least 3 independent experiments and the dots represent the value of each experiment. N.S. , nonsignificant, $P>0.05 ; *, P<0.05 ; * \star, P<0.01 ; * \star \star, P<0.001$. 

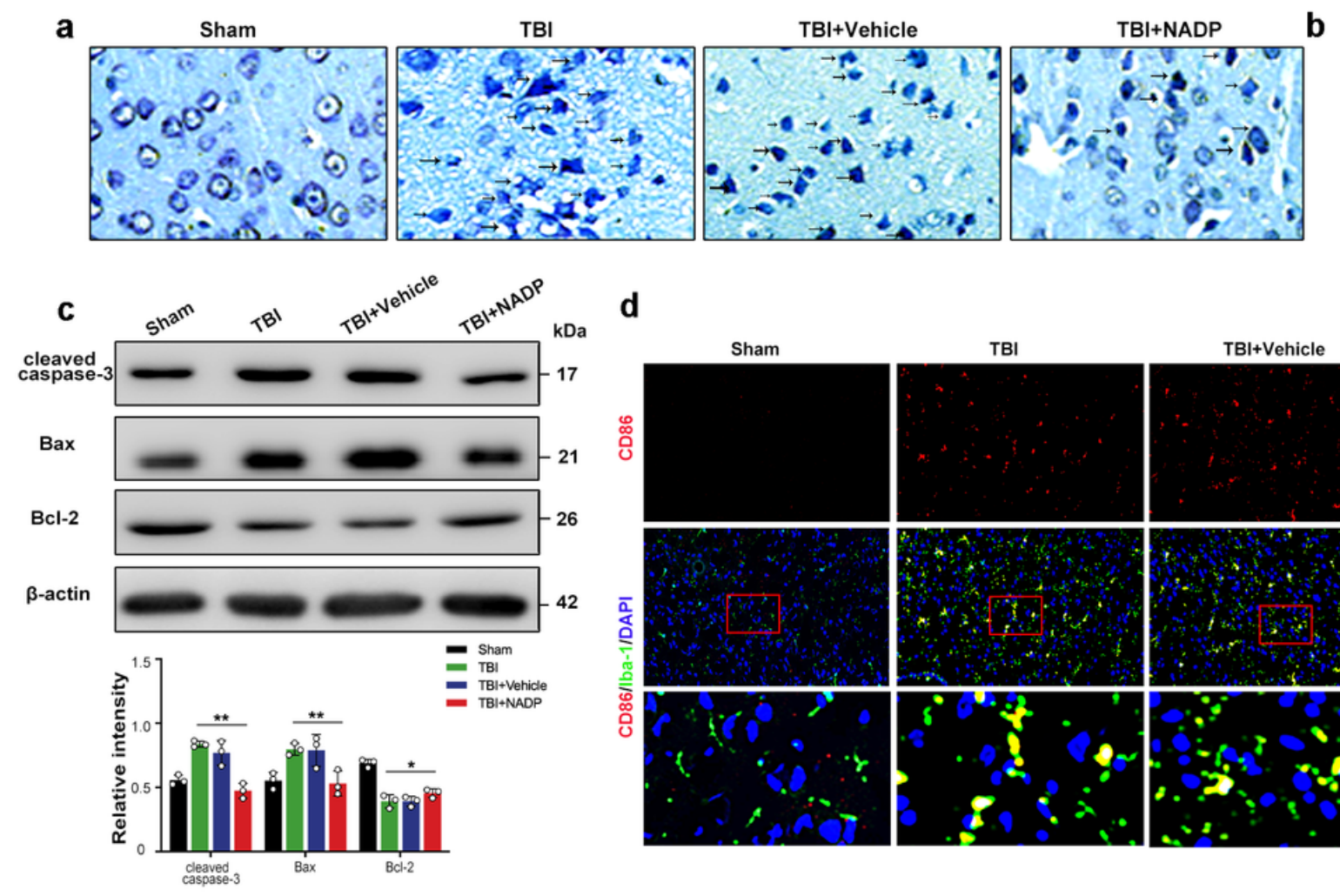

d
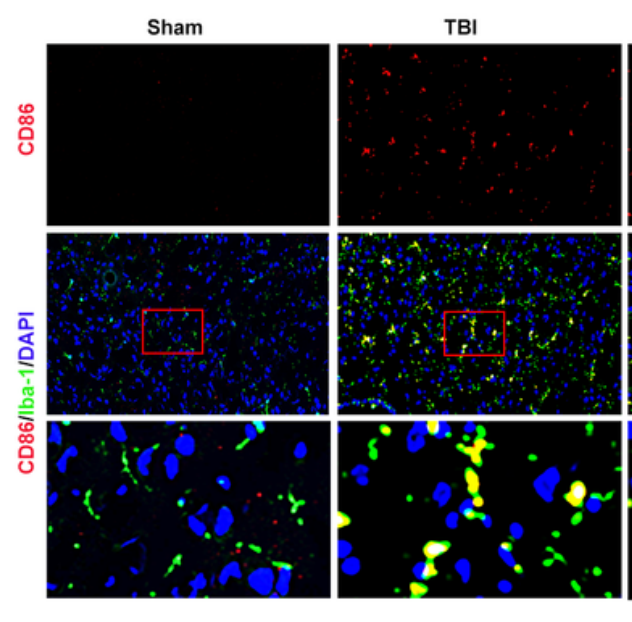
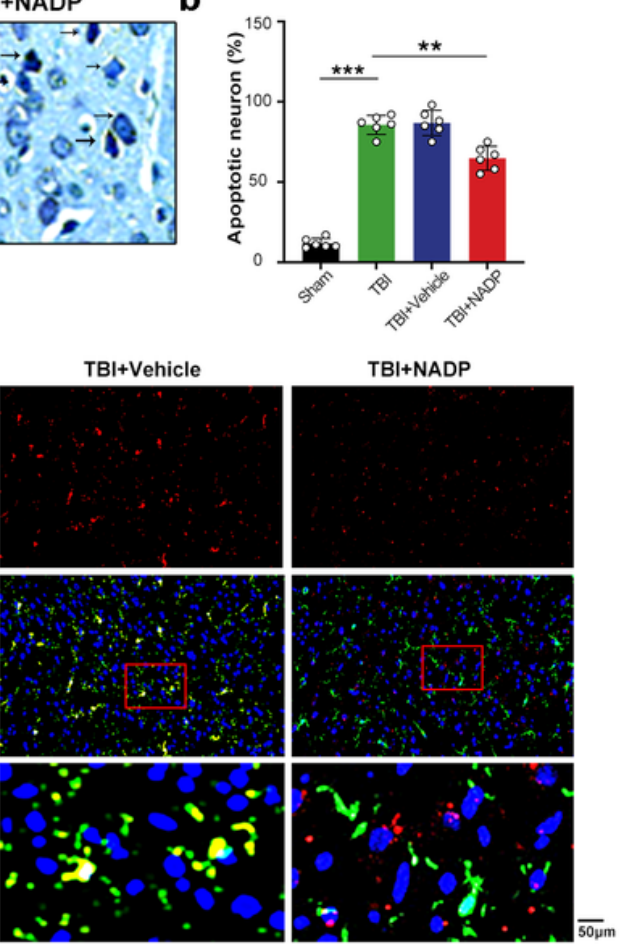
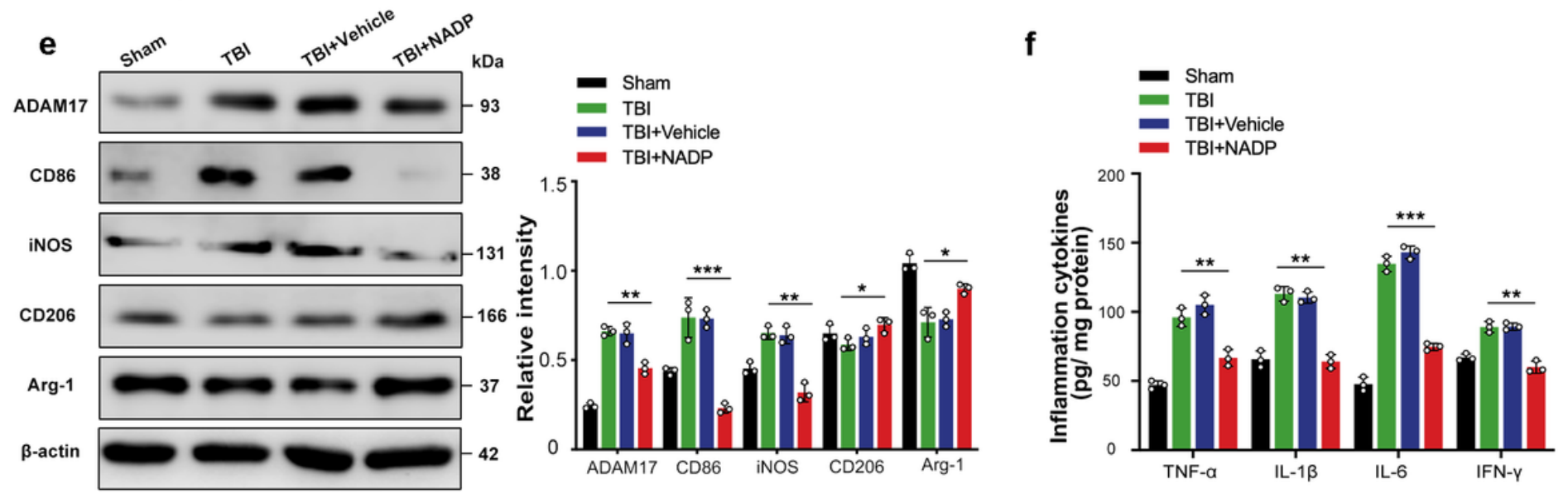

Figure 7

Enhancement of FTO can reduce neuronal apoptosis and inhibit neuroinflammation after TBI in vivo. a, b The percentage of apoptotic cells was higher in the TBI group than in the sham group $(P<0.05)$.

Compared with the TBI group, the neuronal apoptosis rate in the TBI+NADP group was significantly lower at day 3 after TBI $(P<0.05)$. Representative photomicrographs of Nissl-stained neurons are shown. The arrows indicate apoptotic neurons. Scale bars $=50 \mu \mathrm{m}$. c Western blot analyses revealed that TBI resulted in the up-regulation of apoptotic factors in the injured cortex at day 3 after TBI. Compared with the TBI group, the levels of cleaved caspase- 3 and Bax in the TBI+NADP group were decreased, and the antiapoptotic factor Bcl-2 was increased $(P<0.05)$. d Enhancement of FTO redcued M1 (CD86+/lba-1+) microglial polarization. Representative photomicrographs of CD16-positive microglia are shown. Scale bars $=50 \mu \mathrm{m}$. e Compared with the TBI group, the TBI+NADP group had significantly reduced expression of ADAM17, CD86, and iNOS, and increased the expression of CD206 and Arg- $1(P<0.05)$. $f$ ELISA results 
showed that the TBI+NADP group had significantly reduced the expression of TNF- $a$, IL-1 $\beta$, IL- 6 and IFN- $\gamma$, compared with the TBI group $(P<0.05)$. Values are expressed as mean \pm SD from at least 3 independent experiments and the dots represent the value of each experiment. N.S. , nonsignificant, $P>0.05 ; *, P<$ $0.05 ; * \star, P<0.01 ; * \star \star, P<0.001$.

a

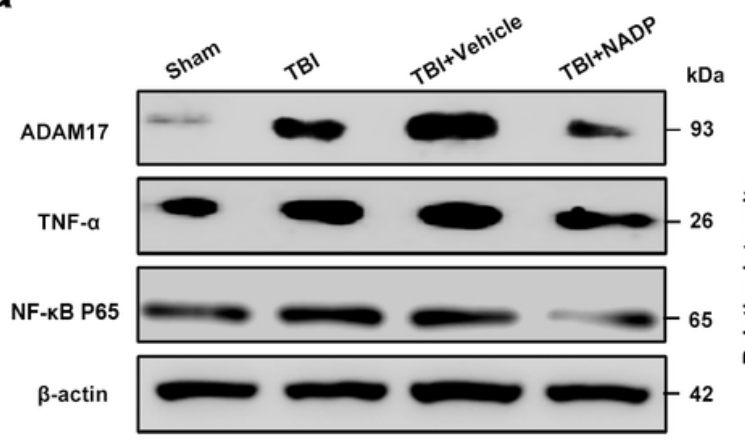

b

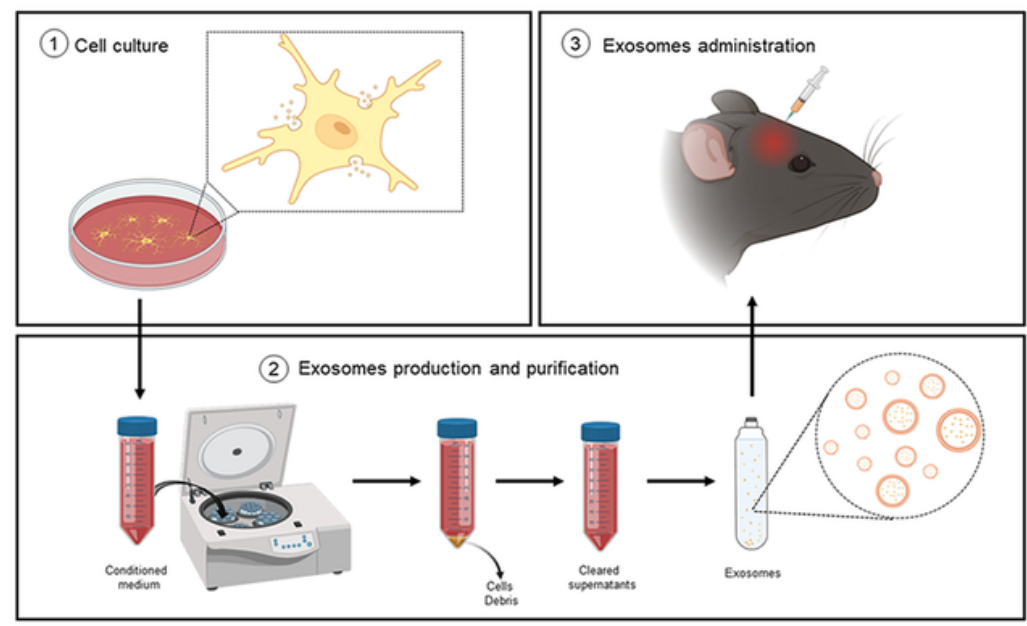

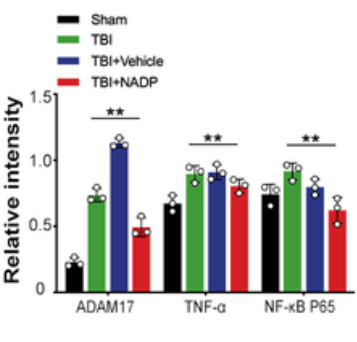

C

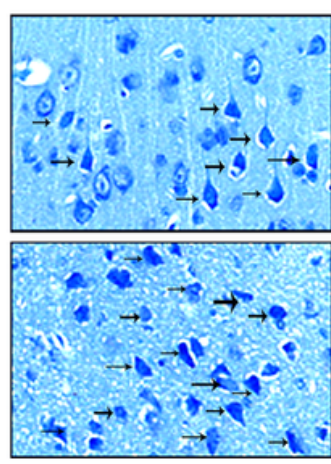

d

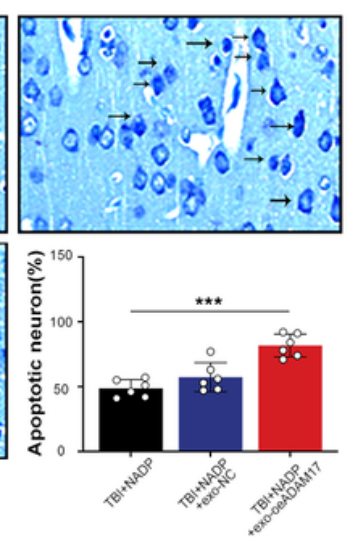

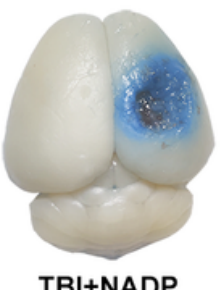

TBI+NADP

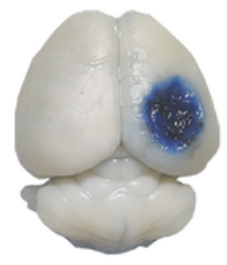

TBI+NADP +exo-oeADAM17
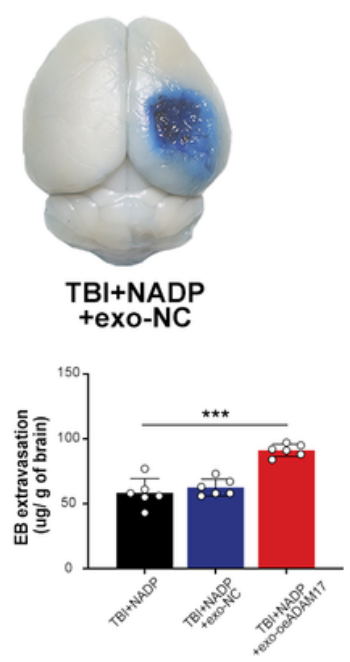
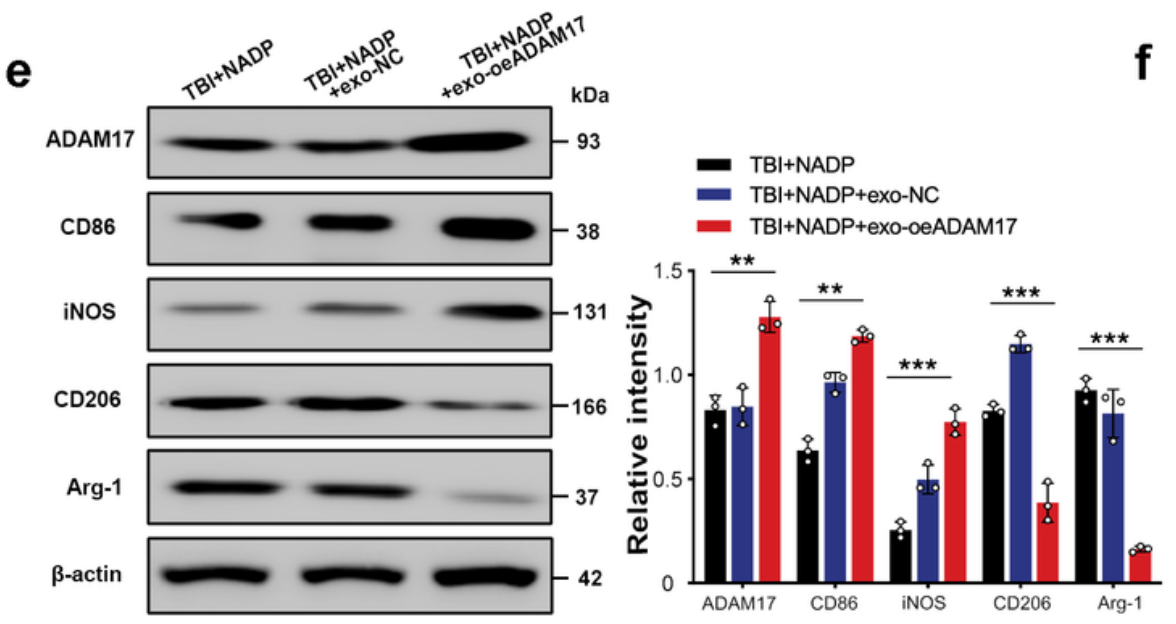

Figure 8 
FTO regulates neuroinflammation in vivo by targeting ADAM17 in microglia after TBI. a Western Blot demonstrated that NADP intervention highly inhibited ADAM17, TNF-alpha, and NF-KB p65 at the protein level. $\mathbf{b}$ Exosomes in the supernatant of ADAM17 overexpression cells were collected for identification, concentration, and quantification. The exosomes were subsequently injected into the damaged cortex. $\mathbf{c}$ The apoptosis rate of neurons in the TBI+NADP+exo-oeADAM17 group was significantly higher than that in the TBI+NADP group at day 3 after TBI $(P<0.05)$. Representative photomicrographs of the Nisslstained neurons are shown. The arrows indicate apoptotic neurons $(P<0.05)$. Scale bars $=50 \mu \mathrm{m}$. $\mathbf{d}$ The brain water content of the TBI+NADP+exo-oeADAM17 group increased significantly at day 3 after TBI $(P<$ 0.05). e Compared with the TBI+NADP group, the TBI+NADP+exo-oeADAM17 group had significantly increased expression of ADAM17, CD86, and iNOS, and decreased expression of CD206 and Arg-1 $(P<$ 0.05). $\mathbf{f}$ ELISA results showed that the TBI+NADP+exo-oeADAM17 group had significantly increased expression of TNF-a, IL-1 $\beta$, IL- 6 and IFN- $\gamma(P<0.05)$. Values are expressed as mean \pm SD from at least 3 independent experiments and the dots represent the value of each experiment. N.S., nonsignificant, $P>$ $0.05 ; *, P<0.05 ; * \star, P<0.01 ; * \star *, P<0.001$.

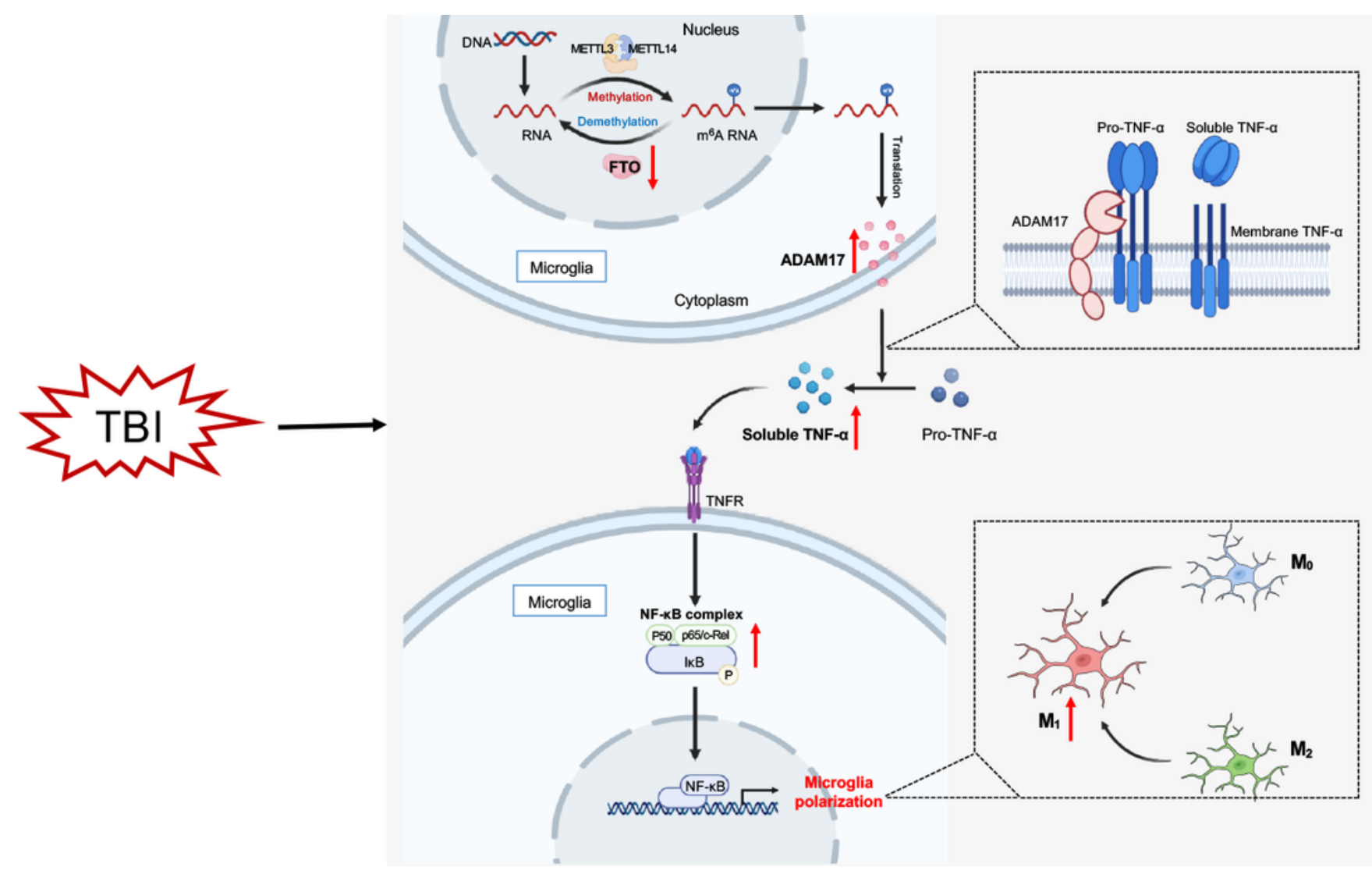

Figure 9

Schematic illustrating the possible mechanisms of FTO in microglial polarization and the neuroinflammatory response after TBI.

As illustrated, FTO, as an important $\mathrm{m}^{6} \mathrm{~A}$ demethylation enzyme, can affect the transcriptional modification of A disintegrin and metalloproteinase 17 (ADAM17). The TNF-a/NF-kB pathway is critical 
to the polarization of the M1 microglial phenotype, which is regulated by the cleavage function of ADAM17. Down-regulation of FTO expression causes abnormally high expression of ADAM17 and downstream TNF- $\alpha$ /NF-kB pathway related factors in microglia, which promote the development of inflammation in the early pro-inflammatory process after TBI.

\section{Supplementary Files}

This is a list of supplementary files associated with this preprint. Click to download.

- Suppl.Fig.1.tif

- Suppl.Table1.xls

- Suppl.Table2.xls

- Suppl.Table3.xls

- Suppl.Table4.xls

- Suppl.Table5.xls 